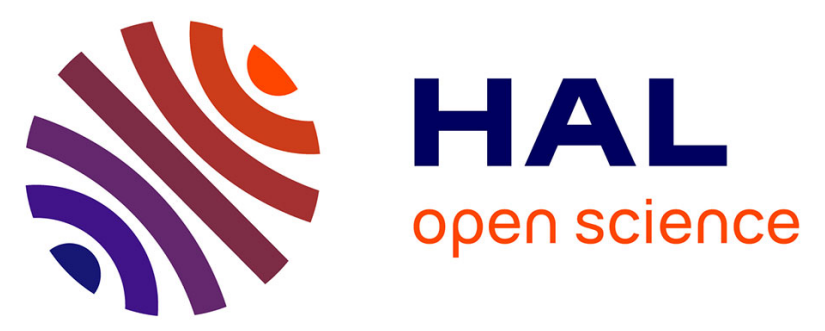

\title{
An arbitrary Lagrangian-Eulerian finite element approach to non-steady state turbulent fluid flow with application to mould filling in casting
}

Laurence Gaston, Alima Kamara, Michel Bellet

\section{- To cite this version:}

Laurence Gaston, Alima Kamara, Michel Bellet. An arbitrary Lagrangian-Eulerian finite element approach to non-steady state turbulent fluid flow with application to mould filling in casting. International Journal for Numerical Methods in Fluids, 2000, 34 (4), p. 341-369. 10.1002/10970363(20001030)34:43.0.CO;2-K . hal-00537933

HAL Id: hal-00537933

https://hal-mines-paristech.archives-ouvertes.fr/hal-00537933

Submitted on 29 Mar 2011

HAL is a multi-disciplinary open access archive for the deposit and dissemination of scientific research documents, whether they are published or not. The documents may come from teaching and research institutions in France or abroad, or from public or private research centers.
L'archive ouverte pluridisciplinaire HAL, est destinée au dépôt et à la diffusion de documents scientifiques de niveau recherche, publiés ou non, émanant des établissements d'enseignement et de recherche français ou étrangers, des laboratoires publics ou privés. 
Int. J. Num. Meth. Fluids 34 (2000) 341-369

\section{AN ARBITRARY LAGRANGIAN EULERIAN FINITE ELEMENT APPROACH TO NON STEADY STATE TURBULENT FLUID FLOW WITH APPLICATION TO MOULD FILLING IN CASTING}

Laurence Gaston, Alima Kamara, Michel Bellet*

Ecole des Mines de Paris

Centre de Mise en Forme des Matériaux (CEMEF)

UMR CNRS 7635

BP 207, 06904 Sophia Antipolis, France

* Correspondence to: Dr Michel Bellet, Ecole des Mines de Paris, CEMEF, BP 207, 06904 Sophia Antipolis, France. Tel: +33 (0) 4939574 61, fax: +33 (0) 4923897 52, e-mail: michel.bellet@cemef.cma.fr 
Abstract

This paper presents a two-dimensional Lagrangian-Eulerian finite element approach of non steady state turbulent fluid flows with free surfaces. The proposed model is based on a velocity-pressure finite element Navier-Stokes solver, including an augmented Lagrangian technique and an iterative resolution of Uzawa type. Turbulent effects are taken into account with the two-equation statistical model $k-\varepsilon$. Mesh updating is carried out through an arbitrary Lagrangian-Eulerian (ALE) method in order to describe properly the free surface evolution. Three comparisons between experimental and numerical results illustrate the efficiency of the method. The first one is a turbulent flow in an academic geometry, the second one is a mould filling in effective casting conditions and the third one is a precise confrontation to a water model.

Keywords: fluid flow - free surface - turbulence - finite element - ALE - mould filling 


\section{Introduction}

From the industrial point of view, the numerical simulation of the mould filling stage of casting processes is carried out following several goals. First, to provide the downstream thermo-mechanical and microstructural computations of the cooling stage with accurate initial conditions in terms of temperature and velocity field. Additionally, to be representative of the fluid flow, in order to understand the occurrence of defects such as incomplete filling due to early solidification of the metal, convection of inclusions, oxidation associated with turbulence, mould wear, etc.

Most mould filling models are of fixed mesh type. This is of course the most convenient way to approach non steady state fluid flow since the computation grid can remain fixed from the beginning to the end of the filling. However in this case, the precision of the computation is fully dependent on the mesh density. This implies that in case of complex flow the user must have a fairly good idea a priori of the liquid flow in order to capture properly the critical features of the flow: jet impact onto a wall, waves, entrapment of air pocket, etc. In addition, one of the main issues in such a formulation is the tracking of the free surface separating the fluid domain from the rest of the cavity. Most $\operatorname{codes}^{1-9}$ use the volume of fluid (VOF) method ${ }^{10}$. It consists in solving the conservation equation $d F / d t=0$ of a variable $F$ whose values are one in filled regions and zero elsewhere. $F$ can be seen as a volumic fraction of fluid and is often called fill factor or pseudoconcentration factor ${ }^{11}$. Two fluids are actually considered: the flowing liquid of interest and a fictitious fluid in the empty regions. This method suffers generally from numerical diffusion in the resolution of the free surface tracking equation, which is of pure advective type. In addition, it is often mentioned that the method can hardly handle the discontinuity of the material viscosity at the interface. To prevent this difficulty, the viscosity value is smoothed around the interface, adding again some imprecision. Since the value $F=1 / 2$ is supposed to represent the free surface, it results in a smeared fluid surface. Another front tracking method is the marker and cell (MAC) method $^{12}$. In this method, some marking particles close to the front are convected with the computed velocity field. The updated location of the free surface is then deduced from the updated locations of the markers. This method requires a great number of markers to be 
precise but have been used in the frame of mould filling simulation ${ }^{13-15}$. Another problem inherent to the fixed mesh methods is that it is difficult to take into account some terms of mechanical loading applied to the fluid surface. This is the case of surface tension effects, which need an explicit determination of the fluid surface as a mesh boundary to be implemented in a proper way ${ }^{16}$.

An alternative to the fixed mesh methods are the methods in which the mesh covers the fluid domain only. The advantage of these moving mesh methods lies in the natural representation of the free surfaces, yielding an accurate description of the front. They can be divided into two categories: updated Lagrangian and Eulerian-Lagrangian methods. In the updated Lagrangian approach, the mesh is convected with the fluid during a given time step (each nodal point is attached to a fluid particle). Hence the mesh encounters enormous distortions during a process such as a mould filling. Remeshing operations are needed very frequently, which is detrimental to the computation time. However, application to mould filling has been carried out ${ }^{17}$. The Arbitrary Lagrangian-Eulerian (ALE) method ${ }^{18-19}$ in which the mesh is updated independently of the material fluid flow is much more flexible. Using this method, the mesh is rearranged at every time increment but keeping the same number of elements and nodes and the same connectivity. Therefore the number of full remeshing operations can be significantly reduced. Hence, in ALE formulation, the nodal velocity field differs from the velocity field of the fluid. This raises two main issues: first the determination of the mesh velocity, and second the treatment of the advection terms arising from this difference. Regarding the first point, the same algorithms as for mesh generation and mesh improvement are used, essentially based on Laplacian regularisation in order to minimise element distortions $\mathrm{s}^{20-21}$ or other node repartition methods ${ }^{22-23}$. The second point is actually not specific to ALE methods, as the same problem must be treated in classical Eulerian formulations. There are two main classes of methods ${ }^{24}$. On one hand, the methods of streamline upwind or streamline upwind Petrov-Galerkin type (SU and SUPG). They consist of a modification of the test functions of the weak form of the advection-diffusion equations. Such methods stabilise the problem by adding some artificial numerical diffusion along streamlines ${ }^{25}$. On another hand, the splitting methods can be used, in which the advectiondiffusion equations are split into a pure advective problem and a pure diffusion problem. The treatment of the advection problem may be of characteristic type. This method consists in 
computing the upstream trajectory of the material particles ${ }^{26}$. A very similar method is the direct interpolation method in which an auxiliary updated Lagrangian configuration is defined allowing the computation of advected values by direct interpolation ${ }^{27}$. This method has been used in the present work and will be detailed in the paper.

Hence the ALE method seems to be promising for mould filling application. However, to our knowledge, its application to casting analysis is still in its infancy. We can quote the work of Lewis et al. ${ }^{16,28}$ and the approach formerly developed by the present authors ${ }^{29,30}$. Both formulations are limited to two-dimensional non turbulent flow. The difference between them lies in the fact that the first one is basically Eulerian (treatment of the advection terms in the momentum equation combined with a Lagrangian free surface incrementation method) whereas the second one is basically Lagrangian (direct time discretisation of total derivative of the velocity in the momentum equation, plus Lagrangian update combined with rezoning). This latest formulation has served as a starting basis for the implementation of turbulence modelling.

The objective of the paper is to present an application of the ALE method in the context of isothermal non steady state turbulent flow. In the next sections, we shall detail the governing equations and boundary conditions, the two-dimensional finite element resolution and the ALE scheme. Then application to mould filling and validation with respect to literature and experimental results obtained on a water model and in casting conditions will be illustrated and discussed.

\section{Governing equations}

\subsection{Incompressible Newtonian fluid}

Molten metallic alloys are assumed incompressible Newtonian fluids. Their governing equations consist of the viscous constitutive equation (1), defining the Cauchy stress tensor with respect to the velocity gradient, and of the incompressibility condition (2).

$$
\begin{gathered}
\boldsymbol{\sigma}=-p \boldsymbol{I}+2 \rho v \dot{\boldsymbol{\varepsilon}} \quad \dot{\boldsymbol{\varepsilon}}=\frac{1}{2}\left(\nabla \boldsymbol{v}+(\nabla \boldsymbol{v})^{T}\right) \\
\operatorname{tr} \dot{\boldsymbol{\varepsilon}}=\nabla \cdot \boldsymbol{v}=0
\end{gathered}
$$


Here, $\sigma$ is the Cauchy stress tensor, $p$ the hydrostatic pressure, $\boldsymbol{I}$ the identity tensor, $\rho$ the specific mass, $v$ the kinematic viscosity of the fluid, $v$ the velocity field, and $\dot{\varepsilon}$ the strain rate tensor.

The local momentum equation is:

$$
\nabla \cdot \sigma+\rho g=\rho \gamma
$$

where $\boldsymbol{g}$ is the gravity and $\gamma$ the acceleration vector. Injection of (1-2) in (3) yields the classical incompressible and isothermal Navier-Stokes equations:

$$
\left\{\begin{array}{l}
\rho \frac{d \boldsymbol{v}}{d t}-\rho \nabla^{2} \boldsymbol{v}+\nabla p=\rho \boldsymbol{g} \\
\nabla \cdot \boldsymbol{v}=0
\end{array}\right.
$$

\subsection{Turbulent fluid}

During mould filling in casting, the metal flow is often turbulent. Most simulation codes ignore the turbulence phenomena. Some of them simply consider an arbitrary increased viscosity. Others include the simple mixing length model based on a definition of viscosity from the norm of average velocity gradients and an arbitrary mixing length related to some characteristic length of the flow. In view of mould filling modelling, we have preferred using the simple but realistic approach to turbulence offered by the statistical turbulence models ${ }^{31}$, and more particularly the two-equation standard $k-\varepsilon$ turbulence model initiated by Launder and Spalding ${ }^{32}$. Like all statistical models, it basically consists of a decomposition of the pressure and velocity fields into a time averaged component (denoted with a bar) and a turbulent fluctuating component (denoted with a prime). For instance, the decomposition of the velocity field $v$ is:

$$
\boldsymbol{v}(\boldsymbol{x}, t)=\overline{\boldsymbol{v}}(\boldsymbol{x}, t)+\boldsymbol{v}^{\prime}(\boldsymbol{x}, t)
$$

where the time averaging is defined as:

$$
\overline{\boldsymbol{v}}(\boldsymbol{x}, t)=\frac{1}{T} \int_{t-T / 2}^{t+T / 2} \boldsymbol{v}(\boldsymbol{x}, \tau) d \tau
$$


$T$ being a time interval assumed large enough with respect to the characteristic time of the fluctuations and small compared to the variation time of the average value. The timeaveraging of the Navier-Stokes equations themselves results then in the following Reynolds equations:

$$
\left\{\begin{array}{l}
\rho \frac{d \overline{\boldsymbol{v}}}{d t}-\rho \nu \nabla^{2} \overline{\boldsymbol{v}}+\rho \nabla \cdot\left(\overline{\boldsymbol{v}^{\prime} \times \boldsymbol{v}^{\prime}}\right)+\nabla \bar{p}=\rho \boldsymbol{g} \\
\nabla \cdot \overline{\boldsymbol{v}}=0
\end{array}\right.
$$

in which we can identify additional Reynolds stresses which are due to turbulent dissipation and whose components are:

$$
R_{i j}=-\rho \overline{v_{i}^{\prime} v_{j}^{\prime}}
$$

According to Boussinesq assumption, the Reynolds stress tensor $\boldsymbol{R}$ can be related to the gradient of the time averaged velocity field of the flow. This is achieved by the so-called turbulent viscosity $v_{T}$, defining a new constitutive equation of the turbulent fluid:

$$
\boldsymbol{R}=\rho v_{T}\left(\nabla \overline{\boldsymbol{v}}+(\nabla \overline{\boldsymbol{v}})^{T}\right)-\frac{2}{3} \rho k \boldsymbol{I}
$$

where $k$ is the turbulent kinetic energy per unit of mass:

$$
k=\frac{1}{2} \overline{v^{\prime} \cdot v^{\prime}}
$$

In the sequel, we shall not use the bar notation any more and $v$ will denote the averaged velocity field in case of turbulent flow. Hence the Navier-Stokes equations (7) are now replaced by:

$$
\left\{\begin{array}{l}
\rho \frac{d \boldsymbol{v}}{d t}-\rho \nabla \cdot\left[\left(v+v_{T}\right) \nabla \boldsymbol{v}\right]+\nabla\left(p+\frac{2}{3} \rho k\right)=\rho \boldsymbol{g} \\
\nabla . \boldsymbol{v}=0
\end{array}\right.
$$

The closure of the turbulent constitutive equations is achieved by defining the evolution law of the turbulent kinematic viscosity. In the $k-\varepsilon$ model, $v_{T}$ depend on the turbulent kinetic energy per unit of mass $k$ and its rate of dissipation, $\varepsilon$ :

$$
v_{T}=C_{\mu} \frac{k^{2}}{\varepsilon}
$$


The variables $k$ and $\varepsilon$ are assumed to obey the two following advection-diffusion equations:

$$
\left\{\begin{array}{l}
\frac{d k}{d t}=\nabla \cdot\left[\left(v+\frac{v_{T}}{\sigma_{k}}\right) \nabla k\right]+C_{\mu} \frac{k^{2}}{\varepsilon} \nabla v:\left(\nabla v+(\nabla v)^{T}\right)-\varepsilon \\
\frac{d \varepsilon}{d t}=\nabla \cdot\left[\left(v+\frac{v_{T}}{\sigma_{\varepsilon}}\right) \nabla \varepsilon\right]+C_{\mu} C_{\varepsilon 1} k \nabla v:\left(\nabla v+(\nabla v)^{T}\right)-C_{\varepsilon 2} \frac{\varepsilon^{2}}{k}
\end{array}\right.
$$

in which $C_{\mu}=0.09, C_{\varepsilon 1}=1.44, C_{\varepsilon 2}=1.92, \sigma_{k}=1.0, \sigma_{\varepsilon}=1.3$, are either empirical or experimentally measured coefficients. The values of these parameters are applied rather universally with the standard $k$ - $\varepsilon$ turbulence model ; alternative values have not been investigated in the frame of this study.

\section{Boundary conditions}

One can refer to figure 1 for the boundary conditions that can be taken into account in the present model. $\Omega$ denotes the domain occupied by the fluid at a current time $t$ of the filling operation (boundary $\partial \Omega$ ).

\subsection{Boundary conditions for laminar Newtonian flow}

- Inlet boundary, $\partial \Omega^{i n j}$

Either a velocity distribution or a uniform pressure can be prescribed. Both can be eventually time dependent. Denoting $\boldsymbol{x}$ the position vector and $\boldsymbol{n}$ the outward unit normal vector to $\partial \Omega^{i n j}$, we have:

$$
\boldsymbol{v}=\boldsymbol{v}^{d}(\boldsymbol{x}, t) \quad \text { or } \quad \boldsymbol{\sigma n}=-P^{d}(t) \boldsymbol{n}
$$

- Free surfaces, $\partial \Omega^{\text {free }}$

A given normal stress vector can be applied:

$$
\boldsymbol{\sigma} \boldsymbol{n}=\boldsymbol{T}^{d}
$$

This vector $\boldsymbol{T}^{d}$, colinear to the outward unit vector $\boldsymbol{n}$, can result from surface tension (modulus proportional to the average surface curvature), or compression of residual gases in 
the cavity. However, the results presented here do not take into account such effects $\left(\boldsymbol{T}^{d}=0\right)$.

- Fluid-mould interface, $\partial \Omega^{\text {mould }}$

A sticking contact condition can be considered $(v=0)$. In practice, the near wall region is often affected by steep velocity gradients and the sticking contact condition can require a very fine mesh near the wall. An alternative consists in using a wall friction law of Newtonian type in which tangential sliding is permitted, the tangential stress vector depending on the tangential velocity of the fluid according to the relation:

$$
\boldsymbol{T}_{f}=-\alpha \rho v \boldsymbol{v}_{t}
$$

In addition the non-penetration condition has to be fulfilled:

$$
\boldsymbol{v} . \boldsymbol{n}=0
$$

\subsection{Boundary conditions for turbulent flow}

- Inlet boundary, $\partial \Omega^{i n j}$

The inlet conditions on velocity and pressure are the same as for laminar flow. In case of prescribed velocity, the values of turbulent variables $k$ and $\varepsilon$ at the inlet can be determined as follows ${ }^{32}$. A certain ratio $\lambda$ of turbulent kinetic energy with respect to the energy of the average flow is assumed, hence yielding the value of $k$ at the inlet. Then a characteristic length of turbulent eddies is chosen, for example a fraction $\mu$ of the inlet diameter $D$. This yields the inlet value of $\varepsilon$ :

$$
k=\lambda\left(v^{d}\right)^{2} \quad \varepsilon=\frac{C_{\mu} k^{3 / 2}}{\mu D}
$$

Typical values $\operatorname{are}^{32} \lambda=0.02$ and $\mu=0.005$.

In case of imposed pressure, similar conditions are prescribed, provided that the order of magnitude of the velocity is known. Otherwise, boundary conditions of Neumann type are assumed:

$$
\nabla k \cdot n=0 \quad \nabla \varepsilon \cdot n=0
$$


- Free surfaces, $\partial \Omega^{\text {free }}$

In addition to (15), Neumann type boundary conditions (19) are assumed for $k$ and $\varepsilon$.

- Fluid-mould interface, $\partial \Omega^{\text {mould }}$

In turbulent flows, the viscous effects remain dominant in near wall zones, so that these regions are always affected by steep velocity gradients. The turbulent wall friction law is defined as follows ${ }^{33}$. The basic idea is to remove the boundary layer from the computational domain and to allow a non zero sliding velocity at this new boundary.

In the near wall region, the tangential velocity profile is governed by:

$$
\frac{\|\boldsymbol{v}\|}{u^{*}}=f\left(y^{+}\right)
$$

The function $f\left(y^{+}\right)$is a function of the dimensionless distance to the wall defined by:

$$
y^{+}=\delta \frac{u^{*}}{v}
$$

where $\delta$ is the actual normal distance to the physical wall and $\mathrm{u}^{*}$ is called the friction velocity and is related to the tangential friction stress $\boldsymbol{T}_{f}$ by:

$$
\left\|\boldsymbol{T}_{f}\right\|=\rho u^{* 2}
$$

Experiments show that the function $f\left(y^{+}\right)$takes the form:

$$
f\left(y^{+}\right)=\frac{1}{\kappa} \ln \left(E y^{+}\right)
$$

where $E$ is a parameter depending on the wall roughness ( $E=9$ for smooth walls). The constant $\kappa$ is the von Karman constant $(=0.41)$.

In the present approach, a simplified version ${ }^{34}$ of the wall function method has been used to approach the dominant viscous effects in the near wall zones, by taking for $y^{+}$a constant value $(=100)$. This value is indeed characteristic of the turbulent sublayer, in which turbulent effects are significant.

The mechanical boundary conditions to be prescribed in this case consist of a friction law and a non-penetration condition: 


$$
\left\{\begin{array}{l}
\boldsymbol{\sigma n}-(\boldsymbol{\sigma n} . \boldsymbol{n}) \boldsymbol{n}=\boldsymbol{T}_{f}=-\rho \frac{\|\boldsymbol{v}\|}{f\left(y^{+}\right)^{2}} v \\
\boldsymbol{v} . \boldsymbol{n}=0
\end{array}\right.
$$

Boundary conditions for turbulent variables $k$ and $\varepsilon$ can then be established ${ }^{31}$ :

$$
k=\frac{u^{*^{2}}}{\sqrt{C_{\mu}}}=\frac{\|v\|^{2}}{\sqrt{C_{\mu}} f\left(y^{+}\right)^{2}} \quad \varepsilon=\frac{u^{*^{3}}}{\kappa \delta}=\frac{\|v\|^{4}}{\kappa v y^{+} f\left(y^{+}\right)^{4}}
$$

\section{Finite element resolution}

\subsection{Time discretisation}

\section{Configuration updating}

Equations (11) are to be solved for the variables velocity and pressure, at each time increment $\Delta t$ of the mould filling simulation. This requires a time integration scheme relating acceleration and velocity fields as well as a time integration scheme for fluid configuration updating.

Let us consider a time increment $[t, t+\Delta t]$, during which the fluid domain evolves from $\Omega^{t}$ to $\Omega^{t+\Delta t}$. The configuration $\Omega^{t}$ and the velocity field $\boldsymbol{v}^{t}$ are assumed to be known. The following scheme is used:

$$
\left\{\begin{array}{c}
\boldsymbol{v}^{t+\Delta t}=\boldsymbol{v}^{t}+\Delta t \boldsymbol{\gamma}^{t+\Delta t}+O\left(\Delta t^{2}\right) \\
\boldsymbol{x}^{t+\Delta t}=\boldsymbol{x}^{t}+\Delta t \boldsymbol{v}^{t}+\frac{\Delta t^{2}}{2} \gamma^{t}+O\left(\Delta t^{3}\right)
\end{array}\right.
$$

A combination of above relations yields the second order accurate time integration scheme, also called Adams-Bashforth scheme (AB2):

$$
\boldsymbol{x}^{t+\Delta t}=\boldsymbol{x}^{t}+\Delta t\left(\frac{3}{2} \boldsymbol{v}^{t}-\frac{1}{2} \boldsymbol{v}^{t-\Delta t}\right)+O\left(\Delta t^{3}\right)
$$

Before giving the resulting incremental resolution scheme, let us comment about the coupling between mechanical and turbulence equations. 


\section{Staggered coupling scheme for mechanical and turbulence resolution}

It should be noted that, as $v_{T}$ and $k$ depend on the velocity field according to (13), the resolution for velocity and pressure and the resolution for the turbulent variables are coupled. However, a staggered coupling algorithm has been developed and has been proven acceptable and efficient when compared with a fully coupled resolution ${ }^{35}$. Hence, when solving the velocity-pressure problem, the turbulent variables are assumed known and fixed, and viceversa. The mechanical problem appears then as a modified Navier-Stokes problem, the nominal viscosity $v$ being replaced by $v+v_{T}$. Regarding pressure, it can be chosen to solve for the auxiliary pressure $p^{\prime}=p+(2 / 3) \rho k$, the actual pressure being recovered $a$ posteriori.

\section{Incremental resolution scheme}

Therefore the incremental resolution scheme consists of the following steps:

- Configuration at time t: $\Omega^{t}, \boldsymbol{x}^{t}, \boldsymbol{v}^{t}, \boldsymbol{v}^{t-\Delta t}, k^{t}, \varepsilon^{t}$ are known.

- Configuration updating: $\Omega^{t+\Delta t}: \boldsymbol{x}^{t+\Delta t}$ is defined according to (27).

- Resolution of (11) for $v^{t+\Delta t}$ and $p^{t+\Delta t}$ on $\Omega^{t+\Delta t}$.

- Resolution of (13) for $k^{t+\Delta t}$ and $\varepsilon^{t+\Delta t}$ on $\Omega^{t+\Delta t}$.

- Updating of variables: $t \leftarrow t+\Delta t, x^{t} \leftarrow x^{t+\Delta t}, \boldsymbol{v}^{t} \leftarrow \boldsymbol{v}^{t+\Delta t}, \boldsymbol{v}^{t-\Delta t} \leftarrow \boldsymbol{v}^{t}$

$$
k^{t} \leftarrow k^{t+\Delta t}, \varepsilon^{t} \leftarrow \varepsilon^{t+\Delta t} .
$$

- Next increment (go back to first step).

\section{Time discretisation of $k$-E equations}

The advection-diffusion equations of the turbulence model are highly coupled and have to be integrated over the time step. Regarding time integration, the Crank-Nicolson time integration scheme has been found appropriate ${ }^{35}$. Hence, taking for instance the equation for $k$, the following quantities are defined:

$$
k^{*}=k^{t+\Delta t / 2}=\frac{k^{t}+k^{t+\Delta t}}{2} \quad \dot{k}^{*}=\left(\frac{d k}{d t}\right)^{t+\Delta t / 2}=\frac{k^{t+\Delta t}-k^{t}}{\Delta t}
$$

The system to be solved is then transformed into: 


$$
\left\{\begin{array}{l}
\frac{2\left(k^{*}-k^{t}\right)}{\Delta t}=\nabla \cdot\left[\left(v+\frac{C_{\mu} k^{*}}{\sigma_{k} \varepsilon^{*}}\right) \nabla k^{*}\right]+C_{\mu} \frac{k^{* 2}}{\varepsilon^{*}} \nabla v:\left(\nabla v+(\nabla v)^{T}\right)-\varepsilon^{*} \\
\frac{2\left(\varepsilon^{*}-\varepsilon^{t}\right)}{\Delta t}=\nabla \cdot\left[\left(v+\frac{C_{\mu} k^{*}}{\sigma_{\varepsilon} \varepsilon^{*}}\right) \nabla \varepsilon^{*}\right]+C_{\mu} C_{\varepsilon 1} k^{*} \nabla v:\left(\nabla v+(\nabla v)^{T}\right)-C_{\varepsilon 2} \frac{\varepsilon^{*^{2}}}{k^{*}}
\end{array}\right.
$$

in which $v$ denotes the latest computed velocity field (i.e. $v^{t+\Delta t}$, according to the previous incremental resolution scheme).

Finally, a linearisation of this set of equations has been achieved by:

- using the values of $k$ and $\varepsilon$ computed at the end of the previous increment in the diffusion and production terms of both equations;

- using the approximation $\varepsilon^{*^{2}} \approx 2 \varepsilon^{t} \varepsilon^{*}-\left(\varepsilon^{t}\right)^{2}$ in the dissipation term of $\varepsilon$;

- substituting $\left(\varepsilon^{t} / k^{t}\right) k^{*}$ for $\varepsilon$ in the equation for $k$ in order to get a better stability, after Koobus ${ }^{36}$.

The final expression of the linearised discrete differential equations is:

$$
\left\{\begin{array}{l}
\frac{2\left(k^{*}-k^{t}\right)}{\Delta t}=\nabla \cdot\left[\left(v+\frac{v_{T}^{t}}{\sigma_{k}}\right) \nabla k^{*}\right]+v_{T}^{t} \nabla v:\left(\nabla v+(\nabla v)^{T}\right)-\frac{\varepsilon^{t}}{k^{t}} k^{*} \\
\left.\frac{2\left(\varepsilon^{*}-\varepsilon^{t}\right)}{\Delta t}=\nabla \cdot\left[\left(v+\frac{v_{T}^{t}}{\sigma_{\varepsilon}}\right) \nabla \varepsilon^{*}\right]+C_{\mu} C_{\varepsilon 1} k^{t} \nabla v:(\nabla v+(\nabla v))^{T}\right)-2 C_{\varepsilon 2} \frac{\varepsilon^{t} \varepsilon^{*}}{k^{t}}+C_{\varepsilon 2} \frac{\left(\varepsilon^{t}\right)^{2}}{k^{t}}
\end{array}\right.
$$

After resolution (see section 4.4), the values at time $t+\Delta t$ are obtained by:

$$
k^{t+\Delta t}=2 k^{*}-k^{t} \quad \varepsilon^{t+\Delta t}=2 \varepsilon^{*}-\varepsilon^{t}
$$

\subsection{Weak form of the mechanical equations}

According to the above mentioned time integration scheme, at each time step, equations (11) have to be solved for $\boldsymbol{v}^{t+\Delta t}$ and $p^{t+\Delta t}$ on the updated geometry $\Omega^{t+\Delta t}$. Let us first define the following spaces:

$$
\begin{aligned}
& H^{1}\left(\Omega^{t+\Delta t}\right)=\left\{q \in L^{2}\left(\Omega^{t+\Delta t}\right) \quad \nabla q \in\left(L^{2}\left(\Omega^{t+\Delta t}\right)\right)^{2}\right\} \\
& \vartheta\left(\Omega^{t+\Delta t}\right)=\left\{\boldsymbol{v} \in\left(H^{1}\left(\Omega^{t+\Delta t}\right)\right)^{2} \mid v=v^{d} \quad \text { on } \partial \Omega_{v}^{t+\Delta t}\right\}
\end{aligned}
$$




$$
\vartheta_{0}\left(\Omega^{t+\Delta t}\right)=\left\{v \in\left(H^{1}\left(\Omega^{t+\Delta t}\right)\right)^{2} \quad \mid \quad v=0 \quad \text { on } \partial \Omega_{v}^{t+\Delta t}\right\}
$$

where $\partial \Omega_{v}$ denotes the part of the boundary where Dirichlet conditions are prescribed on the velocity field (see section 3). $\vartheta$ is the space of "kinematically admissible" velocity fields and $\vartheta_{0}$ is the space of "zero kinematically admissible" velocity fields.

Taking into account the previous time discretisation, the application of the weighted residual method to the above mentioned momentum equations and incompressibility constraint, leads to the following weak form:

$$
\begin{gathered}
\forall \boldsymbol{v}^{*} \in \vartheta_{0} \quad \int_{\Omega^{t+\Delta t}} \rho \frac{1}{\Delta t}\left(\boldsymbol{v}^{t+\Delta t}-\boldsymbol{v}^{t}\right) \cdot \boldsymbol{v}^{*} d V+\int_{\Omega^{t+\Delta t}} 2 \rho\left(v+v_{T}^{t}\right) \dot{\boldsymbol{\varepsilon}}^{t+\Delta t}: \dot{\boldsymbol{\varepsilon}}^{*} d V \\
-\int_{\Omega^{t+\Delta t}} p^{\prime t+\Delta t} \nabla . \boldsymbol{v}^{*} d V-\int_{\partial \Omega^{t+\Delta t}} \boldsymbol{T} \cdot \boldsymbol{v}^{*} d S-\int_{\Omega^{t+\Delta t}} \rho \boldsymbol{g} \cdot \boldsymbol{v}^{*} d V=0 \\
\forall p^{*} \in L^{2}\left(\Omega^{t+\Delta t}\right) \quad \int_{\Omega^{t+\Delta t}} p^{*} \nabla \cdot \boldsymbol{v}^{t+\Delta t} d V=0
\end{gathered}
$$

with

$$
p^{p^{t+\Delta t}}=p^{t+\Delta t}+\frac{2}{3} \rho k^{t}
$$

\subsection{Velocity-pressure finite element formulation}

The fluid domain $\Omega$ is discretised with $\mathrm{P} 2+/ \mathrm{P} 1$ triangular finite elements proposed by Fortin and Fortin ${ }^{37}$ (fig. 2): quadratic interpolation of velocity plus one central node associated with bubble type cubic interpolation function, discontinuous linear pressure. The addition of the central node allows the element to pass the Brezzi-Babuška condition ${ }^{38}$. It can be shown ${ }^{37}$ that the additional degrees of freedom associated with the bubble can be eliminated at the element level, resulting in a simpler treatment similar to the constant pressure $\mathrm{P} 2 / \mathrm{P} 0$ element.

The velocity and pressure fields are interpolated in the following way:

$$
\boldsymbol{v}=\sum_{n=1}^{\text {Nbnoe }} N_{n} \boldsymbol{V}_{n} \quad p=\sum_{k=1}^{\text {Nbpre }} \bar{N}_{k} P_{k}
$$


where Nbnoe and Nbpre are the number of interpolation nodes for velocity (interpolation function $N$ ) and pressure (interpolation function $\bar{N}$ ) respectively.

Injection of finite element interpolations (38) in (35) and (36) results in the following two linear systems, in which $\boldsymbol{V}$ and $\boldsymbol{P}$ are the unknown nodal velocity and pressure vectors (respectively 2.Nbnoe and Nbpre components):

$$
\left\{\begin{array}{l}
\left(\frac{\rho}{\Delta t} \boldsymbol{M}+\boldsymbol{A}\right) \boldsymbol{V}+\boldsymbol{S}^{T} \boldsymbol{P}=\boldsymbol{C} \\
\boldsymbol{S} \boldsymbol{V}=0
\end{array}\right.
$$

where $\boldsymbol{M}$ is the mass matrix and $\boldsymbol{S}$ the matrix of the discrete divergence operator. The components of the different matrices are the following:

$$
\begin{gathered}
A_{\lambda k \mu n}=\int_{\Omega} 2 \rho\left(v+v_{T}\right) \boldsymbol{B}_{\mu n}: \boldsymbol{B}_{\lambda k} d V+\int_{\partial \Omega^{\text {mould }}} \rho \frac{\left\|\boldsymbol{v}^{t}\right\|}{f\left(y^{+}\right)^{2}} N_{n} N_{k} \boldsymbol{\delta}_{\lambda \mu} d S \\
M_{\lambda k \mu n}=\int_{\Omega} N_{k} N_{n} \boldsymbol{\delta}_{\lambda \mu} d V \\
S_{l \mu n}=\int_{\Omega}-\bar{N}_{l} \operatorname{tr}\left(\boldsymbol{B}_{\mu n}\right) d V \\
C_{\lambda k}=\int_{\Omega} \rho g_{\lambda} N_{k} d V+\int_{\partial \Omega^{\text {free }}} T_{\lambda}^{d} N_{k} d S+\int_{\Omega} \frac{\rho}{\Delta t} V_{\lambda n}^{t} N_{n} N_{k} d V
\end{gathered}
$$

In the previous equations, indices $k$ and $n$ vary from 1 to Nbnoe, index $l$ varies from 1 to Nbpre ; indices $\lambda$ and $\mu$ vary from 1 to 2 . The convention of implicit summation of repeated indices is applied, as in the sequel. $\delta$ is the Kronecker symbol and $\boldsymbol{B}$ denotes the discrete differential operator defined by:

$$
\boldsymbol{B}_{\lambda n}=\frac{\partial \dot{\varepsilon}}{\partial V_{\lambda n}} \quad \text { or, in components, } \quad B_{i j \lambda n}=\frac{\partial \dot{\varepsilon}_{i j}}{\partial V_{\lambda n}}=\frac{1}{2}\left(\frac{\partial N_{n}}{\partial x_{j}} \delta_{i \lambda}+\frac{\partial N_{n}}{\partial x_{i}} \delta_{j \lambda}\right)
$$

Equations (39) can be seen as a saddle-point problem of the following Lagrangian: 


$$
L(\boldsymbol{V}, \boldsymbol{P})=\frac{1}{2} \boldsymbol{V}^{T}\left(\frac{\rho}{\Delta t} \boldsymbol{M}+\boldsymbol{A}\right) \boldsymbol{V}+\boldsymbol{V}^{T} \boldsymbol{S}^{T} \boldsymbol{P}-\boldsymbol{V}^{T} \boldsymbol{C}
$$

In practice the augmented Lagrangian $L_{\rho}(\boldsymbol{V}, \boldsymbol{P})$ is used, including an augmentation term resulting from a discrete penalisation of the incompressibility constraint:

$$
L_{\rho}(\boldsymbol{V}, \boldsymbol{P})=L(\boldsymbol{V}, \boldsymbol{P})+\frac{\chi_{p}}{2} \rho\left(v+v_{T}\right) \boldsymbol{V}^{T} \boldsymbol{S}^{T} \overline{\boldsymbol{M}}^{-1} \boldsymbol{S} \boldsymbol{V}
$$

where $\chi_{p}$ is a large positive constant and $\overline{\boldsymbol{M}}$ is the mass matrix associated with pressure interpolation functions:

$$
\bar{M}_{m n}=\int_{\Omega} \bar{N}_{m} \bar{N}_{n} d V
$$

The saddle-point of $L_{\rho}$ is the same as for $L$, but provides higher convergence rates of the Uzawa algorithm described hereunder ${ }^{39}$. The saddle-point of the augmented Lagrangian is reached by solving the following set of equations:

$$
\left\{\begin{array}{l}
\left(\frac{\rho}{\Delta t} \boldsymbol{M}+\boldsymbol{A}+\chi_{p} \rho\left(v+v_{T}\right) \boldsymbol{S}^{T} \overline{\boldsymbol{M}}^{-1} \boldsymbol{S}\right) \boldsymbol{V}+\boldsymbol{S}^{T} \boldsymbol{P}=\boldsymbol{C} \\
\boldsymbol{S} \boldsymbol{V}=0
\end{array}\right.
$$

The Uzawa's algorithm is based on the following iterative velocity and pressure corrections:

- Initialisation: $i=0$, initial $\boldsymbol{P}^{(0)}$

- Iteration $(i): i=i+1$

- Step 1/ velocity resolution:

$$
\boldsymbol{V}^{(i)}=\left[\frac{\rho}{\Delta t} \boldsymbol{M}+\boldsymbol{A}+\chi_{p} \rho\left(v+v_{T}\right) \boldsymbol{S}^{T} \overline{\boldsymbol{M}}^{-1} \boldsymbol{S}\right]^{-1}\left(\boldsymbol{C}-\boldsymbol{S}^{T} \boldsymbol{P}^{(i-1)}\right)
$$

- $\quad$ Step 2/ pressure correction:

$$
\boldsymbol{P}^{(i)}=\boldsymbol{P}^{(i-1)}+\omega \rho\left(v+v_{T}\right) \overline{\boldsymbol{M}}^{-1} \boldsymbol{S} \boldsymbol{V}^{(i)}
$$

- Repeat iteration until convergence:

$$
\left\|\boldsymbol{S} \boldsymbol{V}^{(i)}\right\|<\boldsymbol{\varepsilon}_{c}
$$


The resolution of the system (49) is performed by a Crout direct elimination method. It should be noted that only one $L D U$ decomposition is needed (achieved at the first iteration). The convergence is generally reached within 4 iterations. Usually, we take $\omega=\chi_{p}=10^{6}$. Thanks to the discontinuous pressure discretisation, the matrix $\overline{\boldsymbol{M}}$ can be inverted at the element level, before assembling. In the limit case where the pressure field is constant per element (P0 approximation), which is achieved after elimination of bubble degrees of freedom, $\overline{\boldsymbol{M}}$ is a diagonal matrix, the $e^{\text {th }}$ term being the volume of element $e$. Therefore this formulation is quite appropriate and efficient.

\subsection{Finite element resolution of turbulence equations}

The P2-Lagrange six-node quadratic interpolation is used to discretise the turbulent variables $k$ and $\varepsilon$ :

$$
k=\sum_{n=1}^{\text {Nbnoe }} N_{n} k_{n} \quad \varepsilon=\sum_{n=1}^{\text {Nbnoe }} N_{n} \varepsilon_{n}
$$

The application of the Galerkin formulation to (30) yields the following sets of equations:

$$
\left\{\begin{array}{l}
\boldsymbol{H}^{k} \boldsymbol{K}^{*}=\boldsymbol{F}^{k} \\
\boldsymbol{H}^{\varepsilon} \boldsymbol{E}^{*}=\boldsymbol{F}^{\varepsilon}
\end{array}\right.
$$

where $\boldsymbol{K}^{*}$ and $\boldsymbol{E}^{*}$ are the global vectors whose components are the nodal values of variables $k^{*}$ and $\varepsilon^{*}$ respectively. Matrices $\boldsymbol{H}$ and vectors $\boldsymbol{F}$ have the following expression:

$$
\begin{gathered}
H_{i j}^{k}=\int_{\Omega}\left(v+\frac{v_{T}^{t}}{\sigma_{k}}\right) \frac{\partial N_{i}}{\partial x_{\lambda}} \frac{\partial N_{j}}{\partial x_{\lambda}} d V+\int_{\Omega}\left(\frac{2}{\Delta t}+\frac{\varepsilon^{t}}{k^{t}}\right) N_{i} N_{j} d V \\
H_{i j}^{\varepsilon}=\int_{\Omega}\left(v+\frac{v_{T}^{t}}{\sigma_{\varepsilon}}\right) \frac{\partial N_{i}}{\partial x_{\lambda}} \frac{\partial N_{j}}{\partial x_{\lambda}} d V+\int_{\Omega}\left(\frac{2}{\Delta t}+2 C_{\varepsilon 2} \frac{\varepsilon^{t}}{k^{t}}\right) N_{i} N_{j} d V \\
F_{i}^{k}=\int_{\Omega}\left(\frac{2 k^{t}}{\Delta t}+v_{T}^{t} \nabla \boldsymbol{v}:\left[\nabla \boldsymbol{v}+(\nabla \boldsymbol{v})^{T}\right]\right) N_{i} d V
\end{gathered}
$$




$$
F_{i}^{\varepsilon}=\int_{\Omega}\left(C_{\mu} C_{\varepsilon 1} k^{t} \nabla \boldsymbol{v}:\left[\nabla \boldsymbol{v}+(\nabla v)^{T}\right]+C_{\varepsilon 2} \frac{\left(\varepsilon^{t}\right)^{2}}{k^{t}}+\frac{2 \varepsilon^{t}}{\Delta t}\right) N_{i} d V
$$

At this stage it should be noted that the variables $k$ and $\varepsilon$ are positive. However, the combination of time integration and numerical discretisation can result in too small or even negative solutions. In order to remedy to such problems, the values are filtered and corrected according to the following rules. The strategy is similar to the one developed by Pelletier et $a l^{40}$ :

- A lower bound for $k$ is defined, corresponding to a fraction $\alpha_{k}$ of the current maximum turbulent kinetic energy:

$$
\text { if } k<k_{\min \_ \text {crit }} \text { then } k=\alpha_{k} k_{\max }
$$

- The dissipation rate $\varepsilon$ is chosen such that the turbulent viscosity should be a fraction $\alpha_{v}$ of the laminar viscosity $v$.

$$
\text { if } \varepsilon<\varepsilon_{\text {crit }} \text { then } \varepsilon=\frac{C_{\mu} k^{2}}{\alpha_{\nu} \nu}
$$

\section{Arbitrary Eulerian-Lagrangian formulation}

\subsection{Mesh updating}

The updating of fluid particles is defined by (26) and (27). In a Lagrangian finite element method, each node $n$ of the dicretisation of the fluid domain $\Omega$ should be updated with the following time integration scheme:

$$
\boldsymbol{X}_{n}^{t+\Delta t}=\boldsymbol{X}_{n}^{t}+\Delta t \boldsymbol{V}_{n}^{t}+\frac{\Delta t^{2}}{2} \boldsymbol{\Gamma}_{n}^{t}=\boldsymbol{X}_{n}^{t}+\Delta t\left[\frac{3}{2} \boldsymbol{V}_{n}^{t}-\frac{1}{2} \boldsymbol{V}_{n}^{t-\Delta t}\right]=\boldsymbol{X}_{n}^{t}+\boldsymbol{U}_{n}^{m a t}
$$

where $\boldsymbol{U}_{n}^{\text {mat }}$ denotes the material incremental displacement of node $n$.

As mentioned earlier, in a ALE formulation, the displacement of the nodes differ from the displacement of the material particles. In the present ALE formulation, the nodes are displaced incrementally with mesh displacements $\boldsymbol{U}^{m s h}$ which do not depend on the fluid particles displacements $\boldsymbol{U}^{\text {mat }}$, except on the free surface, as explained hereunder. 


\section{Update of internal nodes}

The updating procedure of internal nodes is based on a mesh regularisation method initially developed by Magnin ${ }^{23}$. The incremental displacements of apex nodes (a quadratic triangle has three apex nodes and three mid-edge nodes) are calculated in order to minimise the distortion of the updated mesh. This can be achieved by writing that, after updating, each internal apex node $n$ should be as close as possible to the centre of gravity of the polygon joining the centres of gravity of elements $e$ surrounding the node $n$. Denoting $n b e(n)$ the number of elements surrounding $n$ and assuming for simplicity that surrounding elements are numbered from 1 to $n b e(n)$, this can be expressed as follows:

$$
\boldsymbol{X}_{n}^{t+\Delta t}=\frac{1}{n b e(n)} \sum_{e=1}^{n b e(n)} \sum_{m \in e} \frac{1}{3} \boldsymbol{X}_{m}^{t+\Delta t}
$$

in which the last sum is extended over the three apex nodes $m$ of element $e$. Taking into account the fact that the number of neighbouring apex nodes $n b v(n)$ is equal to the number of surrounding elements and that each neighbouring apex node belongs to two surrounding elements, this can be rewritten in terms of neighbouring apex nodes:

$$
\boldsymbol{X}_{n}^{t+\Delta t}=\frac{1}{n b v(n)} \sum_{v=1}^{n b v(n)}\left(\frac{1}{3} \boldsymbol{X}_{n}^{t+\Delta t}+\frac{2}{3} \boldsymbol{X}_{v}^{t+\Delta t}\right)=\frac{1}{3} \boldsymbol{X}_{n}^{t+\Delta t}+\frac{1}{n b v(n)} \sum_{v=1}^{n b v(n)} \frac{2}{3} \boldsymbol{X}_{v}^{t+\Delta t}
$$

Introducing now the incremental mesh displacement, we have:

$$
\boldsymbol{X}_{n}^{t}+\boldsymbol{U}_{n}^{m s h}=\frac{1}{3}\left(\boldsymbol{X}_{n}^{t}+\boldsymbol{U}_{n}^{m s h}\right)+\frac{2}{3 n b v(n)} \sum_{v=1}^{n b v(n)}\left(\boldsymbol{X}_{v}^{t}+\boldsymbol{U}_{v}^{m s h}\right)
$$

The regularisation is then performed on the basis of this relation. The procedure is iterative of Jacobi type and consists in computing at each iteration $(v)$ the estimates of the nodal mesh displacements in the following way:

$$
\boldsymbol{U}_{n}^{m s h(v+1)}=-\frac{2}{3} \boldsymbol{X}_{n}^{t}+\frac{1}{3} \boldsymbol{U}_{n}^{m s h(v)}+\frac{2}{3 n b v(n)} \sum_{v=1}^{n b v(n)}\left(\boldsymbol{X}_{v}^{t}+\boldsymbol{U}_{v}^{m s h(v)}\right)
$$


This is repeated up to stagnation (i.e. minor relative corrections of the nodal displacements) and the updating scheme of internal apex nodes is then applied, providing the definition of the mesh velocity vectors (here $\Delta t_{2}$ is denoted $\Delta t$ for the sake of simplicity):

$$
\boldsymbol{X}_{n}^{t+\Delta t}=\boldsymbol{X}_{n}^{t}+\boldsymbol{U}_{n}^{m s h(\infty)}=\boldsymbol{X}_{n}^{t}+\Delta t \boldsymbol{V}_{n}^{m s h}
$$

\section{Update of free surface nodes}

The same kind of regularisation is applied on the free surface, with the additional constraint that the incompressibility of the fluid should be preserved, i.e. the mesh velocity field should give rise to the same material flux through the free surface as the material velocity field. Rewriting (60) as $\boldsymbol{X}_{n}^{t+\Delta t}=\boldsymbol{X}_{n}^{t}+\Delta t \boldsymbol{V}_{n}^{\text {mat }}$, the conservation condition is approached by the fulfilment of the following condition by the mesh velocity vector of each node $n$ of the free surface, as illustrated by figure 3 :

$$
\boldsymbol{V}_{n}^{m s h} \cdot \boldsymbol{n}_{n}^{t+\Delta t}=\boldsymbol{V}_{n}^{m a t} \cdot \boldsymbol{n}_{n}^{t+\Delta t}
$$

For nodes located at angular points of the free surface, the mesh velocity is prescribed equal to the material velocity field (fig. 3). For other nodes, (66) determines the normal components of $\boldsymbol{V}^{m s h}$. In the tangent direction, the previous regularisation technique is applied by projection of (61):

$$
\Delta t \boldsymbol{V}_{n}^{m s h(v+1)} \cdot \boldsymbol{t}_{n}^{(v)}=\left[\frac{1}{2}\left(\boldsymbol{X}_{n-}^{t}+\boldsymbol{X}_{n+}^{t}\right)-\boldsymbol{X}_{n}^{t}\right] \cdot \boldsymbol{t}_{n}^{(v)}+\frac{\Delta t}{2}\left(\boldsymbol{V}_{n-}^{m s h(v)}+\boldsymbol{V}_{n+}^{m s h(v)}\right) \cdot \boldsymbol{t}_{n}^{(v)}
$$

Here, $\boldsymbol{X}_{n-}$ and $\boldsymbol{X}_{n+}$ denote the position vectors of the two neighbours of node $n$ on the free surface, $\boldsymbol{t}_{n}$ is the unit tangent vector at node $n$, associated with $\boldsymbol{n}_{n}$.

Finally, after all internal apex nodes and free surface nodes have been displaced, the position of internal mid-edge nodes is determined by the relation:

$$
\boldsymbol{X}_{m / n}^{t+\Delta t}=\frac{1}{2}\left(\boldsymbol{X}_{m}^{t+\Delta t}+\boldsymbol{X}_{n}^{t+\Delta t}\right)
$$

It should be noted that the mesh updating described above enables to delay mesh degeneracy but cannot avoid it totally. Should elements be too distorted, a completely new 
mesh is built using an automatic remeshing technique ${ }^{41}$. This mesh generator has been improved by Glut ${ }^{42}$ to deal with self-contact which often occurs in casting: breaking waves, flow around obstacles.

\subsection{Transport phase}

In the frame of the ALE formulation, the total derivative of any material variable $B$ can be expressed by:

$$
\frac{d B}{d t}=\left.\frac{\partial B}{\partial t}\right|_{m s h}+\nabla B .\left(v^{m a t}-v^{m s h}\right)
$$

where the partial derivative in the right-hand side denotes the rate of variation of $B$ at a given point of the mesh. As already said in introduction, in the literature, this expression is frequently directly implemented in the weak form of the mechanics, turbulence and heat transfer equations. Streamline upwind (SU) or streamline upwind - Petrov-Galerkin (SUPG) techniques are then used, stabilising the advective terms by use of artificial diffusion along streamlines ${ }^{25}$.

Alternative methods consist in addressing directly the material derivative of variable $B$. This requires at each nodal point $n$, of coordinate vector $x^{t+\Delta t}$, the determination of the value of $B$, at previous time $t$, for the particle located at $\boldsymbol{x}^{t+\Delta t}$ at time $t+\Delta t$. This value is denoted $B^{m a t, t}\left(\boldsymbol{x}^{t+\Delta t}\right)$. A first order backward Euler scheme on the particle trajectory during the time increment $[t, t+\Delta t]$ provides the following approximation of the material derivative:

$$
\frac{d B}{d t} \approx \frac{1}{\Delta t}\left(B^{t+\Delta t}\left(\boldsymbol{x}^{t+\Delta t}\right)-B^{m a t, t}\left(\boldsymbol{x}^{t+\Delta t}\right)\right)
$$

The calculation of $B^{\text {mat,t }}\left(\boldsymbol{x}^{t+\Delta t}\right)$ can be achieved using, for instance, a characteristic method $^{43,26,23}$. In the present study, a "pseudo Lagrangian" method has been adopted (fig. 4). In this method, an auxiliary Lagrangian update $\Omega_{L}^{t+\Delta t}$ of the configuration is used. It is defined by (60). Considering the position $x^{t+\Delta t}$, the associated element $e$ of $\Omega_{L}^{t+\Delta t}$ and local coordinates $(\xi, \eta)$ in this element are determined. Then, since $\Omega_{L}^{t+\Delta t}$ is a material update of $\Omega^{t}$, the value $B^{m a t, t}\left(\boldsymbol{x}^{t+\Delta t}\right)$ is computed by direct interpolation in element $e$ of $\Omega^{t}$ :

$$
B^{m a t, t}\left(\boldsymbol{x}^{t+\Delta t}\right)=N_{n}(\xi, \eta) B_{n}^{t}
$$


This technique is applied to determine the following quantities:

- nodal values $k^{t}$ and $\varepsilon^{t}$ of variables $k$ and $\varepsilon$ of turbulence discrete equations (30);

- nodal velocity vectors $\boldsymbol{V}^{t}$ needed in discrete equilibrium equations (48).

\section{Validation of the model on an academic turbulent flow}

The backward facing step test ("BFS") is a classical test often used in the literature for validation of turbulence models. As it involves complex physical phenomena, such as wall reattachment of sheared layers, it is a discriminant test for evaluating the performance and limitations of numerical models. In addition, experimental results are available in the literature.

The test configuration and the mesh which has been used in the present study are presented at figure 5. In order to compare with the experimental measurements of Westphal et $a l .^{44}$, obtained for a nominal Reynolds number equal to 42000 , the following parameters have been used :

- kinematic viscosity of the fluid (air)

$$
\begin{aligned}
& v=1.410^{-5} \mathrm{~m}^{2} . \mathrm{s}^{-1} \\
& H=0.0508 \mathrm{~m} \\
& V_{a v}=11.562 \mathrm{~m} \cdot \mathrm{s}^{-1}
\end{aligned}
$$

- step height

- inlet average velocity

A parabolic velocity profile has been prescribed at the inlet $\left(V_{\min }=10.686 \mathrm{~m} \cdot \mathrm{s}^{-1}, V_{\max }=\right.$ $12.0 \mathrm{~m} \cdot \mathrm{s}^{-1}$ ), with values of $k$ and $\varepsilon$ as indicated by (18) with $v^{d}=V_{a v}$ and $D=3 H / 2$.

Along the lateral walls, the turbulent wall friction law has been used. The parameter $y^{+}$ has been fixed to 100 on the whole fluid-wall interface. At the outlet, a Neumann natural condition is applied to $v, k, \varepsilon$.

Using the present ALE formulation, which is essentially non steady state, the steadystate regime of the test is reached after convergence of a series of time steps $\Delta t=2.10^{-4} \mathrm{~s}$, during which the fluid domain is not updated. The convergence is obtained after 1300 time steps (2h30 CPU on a IBM/RS6000/390), yielding a relative precision on the velocity field equal to $8.10^{-5}$. The figure 6 illustrates the stream lines at convergence, showing the recirculation loop behind the step.

The results have been compared to the experimental measurements of Westphal et al. The figure 7 summarises this comparison. On this figure, the normalised horizontal velocity 
$\hat{U}_{x}=v_{x} / V_{a v}$ has been plotted versus the normalised vertical coordinate $\hat{y}=y / H$, in six transverse sections located at various normalised horizontal coordinates : $\hat{x}=x / H$. It can be seen that a good agreement is obtained with respect to experimental measurements and to other results taken from the literature ${ }^{45}$. The position of the reattachment point is slightly underestimated by the model ( $x=6 H$, instead of $x=7 H$ experimentally). This is a wellknown result for the standard $k-\varepsilon$ model $^{45,46}$.

\section{Application to mould filling and experimental comparison}

\subsection{Casting experiment}

We present first an experimental work, performed by PSA Peugeot-Citroën, on a threedimensional mould equipped with contact sensors. This test has served as a first confrontation test of the numerical model. As shown on figure 8 , the geometry presents some significant difficulties regarding flow simulation: a thin runner $(8 \times 8 \mathrm{~mm}$ in its vertical part) with a thicker zone in its horizontal part ; a backward facing step at the outlet of the runner, causing a free fall of the liquid jet into the mould; a core located in the middle of the cavity, which is an obstacle to the flow. Except the funnel-shaped ingate and the riser, the mould has a constant thickness of $8 \mathrm{~mm}$, so that the flow can be analysed with a two-dimensional model.

An operator pours liquid grey iron in the funnel-shaped pouring gate which is kept filled up until the end of the filling. The metal flows down (by gravity) in the runner, then drops down into the cavity, and fills it progressively. The contact sensors are activated when the metal touches them (and remain so the rest of the time). Activation times are recorded, and the flow advance can be approximately tracked this way (see fig. 9, first line).

In order to compare our two-dimensional simulation with the experiment, we focus the study on the constant thickness part of the experimental mould, e.g. the runner and the cavity. The pouring gate, in which complex flow occurs, because of its variable thickness, is represented by a simple feeding channel, with prescribed pressure, in the simulation (fig. 8). Of course this causes a certain loss of accuracy. As the riser thickness is much bigger than the cavity one, the simulation stops at the riser inlet (further comparison would be irrelevant). 
Because the present finite element model cannot take into account yet thermal coupling in turbulent conditions, we have assumed isothermal laminar conditions. The following data have been used:

- kinematic viscosity

$$
\begin{aligned}
& \nu=2.4610^{-4} \mathrm{~m}^{2} \cdot \mathrm{s}^{-1} \\
& \rho=5695 \mathrm{~kg} \cdot \mathrm{m}^{-3} \\
& \alpha=0.3 \mathrm{~mm}^{-1}
\end{aligned}
$$

- specific mass

- Newtonian friction coefficient

- external pressure applied at inlet surface and free surface $\quad P^{d}=0 \mathrm{~Pa}$

The viscosity has been multiplied by a factor 100 with respect to the real one, in order to simulate the turbulence effect with the laminar type model (the nominal Reynolds number in the runner is about 2000). The friction coefficient has been chosen, according to the observed metal velocity in the runner.

The simulation has needed 2850 time steps, 250 remeshings and took 32 hours CPU on a IBM/RS6000/390. Globally, the results are in good agreement with the experiment, as shown on figure 9. The saturation of the runner seems to be well simulated, as well as the liquid jet coming out of the runner. However, regarding this last point, we can see from the second figure of activated sensors (at time $0.78 \mathrm{~s}$ ) that they seem to indicate a thicker jet shape. The reason for this is not clear and it is possible that the sensor indication has been perturbed by some splashing or wetting of the walls by the jet. Anyway, the simulated flow is in good agreement with the measurements for the rest of the filling: the evolution of the free surface is correct and the filling time as well.

\subsection{Experiment with a water model}

In order to go further the previous encouraging results and to have a deeper insight into the capacity of the model to describe the fluid flow accurately, the formulation has been compared to a water model, in which the experimental flow can be easily observed through a transparent mould made of plexiglas ${ }^{\circledR}$. The experimental device is shown on figure 10 . A similar shape has been chosen, but of different dimensions : the square cavity is $320 \mathrm{x} 320$ $\mathrm{mm}$ and the thickness has been taken equal to $20 \mathrm{~mm}$. The experiments have been recorded with a numerical video recorder.

The simulation has been carried out using the turbulence $k$ - $\varepsilon$ model. As the experimental flow rate during the beginning of the mould filling corresponds to an average 
velocity of $0.875 \mathrm{~m} . \mathrm{s}^{-1}$ in the runner, yielding a nominal Reynolds number of about 20000 , the inlet values of $k$ and $\varepsilon$ have been prescribed as indicated by (18): $k=1.510^{-2} \mathrm{~m}^{2} \cdot \mathrm{s}^{-2}$ and $\varepsilon$ $=4.1 \mathrm{~m}^{2} \cdot \mathrm{s}^{-3}$. Regarding the wall law, the parameter $y^{+}$has been fixed to 100 .

The figure 11 shows a typical comparison between the computed and observed evolution of the free surface. Globally, the agreement is excellent. One of the main differences is the jet orientation. Unlike in the laminar simulation presented above, the wall law parameter has not been deduced from the experiment since a fixed value has been used (100). It is worth noting that the predicted jet velocity is obviously not very far from the real one. This validates a posteriori the choice of this value, since a lower value would have increased the friction effects and, since the runner is quite narrow, it would have resulted in a lower predicted jet velocity. We can notice also that the enlargement of the jet - already observed in the casting experiment - is present in the real flow, whereas the simulation does not render this effect (which could be due again to wetting of the plexiglas ${ }^{\circledR}$ mould by water).

On figure 12 , the isovalues of the ratio $v_{T} / v$ are plotted, showing maximum values around 100, corresponding to the regions where the velocity gradients are maximum. It can be seen, from the distribution of the turbulent kinetic energy $k$ at different times, that the turbulence decreases rapidly when the cavity fills up, especially when the core and the inlet jet become submerged. At this stage, the use of the standard $k-\varepsilon$ model, which is normally applicable to fully developed turbulent flows, is less and less valid. This is confirmed in the computation by more and more occurrences of negative values of $k$, needing arbitrary correction techniques (eq. (58) and (59)). Obviously, in such conditions low Reynolds $k$ - $\varepsilon$ models and an approach to the laminar transition should be used. This is a natural extension to this work.

Finally, we can comment on the comparison between laminar and turbulent computations. In the present case, both approaches have been used and a detailed comparison can be found elsewhere ${ }^{35}$. Globally, the results are similar and it is difficult to determine which formulation is better. However, the merit of the turbulent computation is to avoid the guess of an arbitrary viscosity in order to stabilise the laminar flow model.

\section{Conclusion}


In this paper, a two-dimensional finite element formulation for non steady state turbulent fluid flows with free surface has been presented. It is based upon an ALE NavierStokes solver including the coupling with the turbulence $k-\varepsilon$ model. Comparisons with reference literature results and with experimental results, obtained both in casting condition and on a water model, have shown the efficiency of this approach.

Regarding application to the simulation of casting processes, the next improvements should be the implementation of the thermal coupling, allowing the computation of the temperature field in the fluid and in the mould. Further work will deal with the enrichment of the physical model used to describe fluid flow: implementation of surface tension and wall adhesion, use of low Reynolds $k-\varepsilon$ model. Finally, a three-dimensional extension of the present approach should be developed.

\section{Acknowledgement}

The authors would like to thank Creusot-Loire Industrie (CLI), The French Ministère de l'Enseignement Supérieur et de la Recherche and Agence de l'Environnement et de la Maîtrise de l'Energie (Ademe) for their financial support. They are also deeply indebted to PSA Peugeot-Citroën for the experimental work and to all the participants of the working group on mould filling organised by the French Centre Technique des Industries de la Fonderie (CTIF) for fruitful discussions. 


\section{References}

1. G.P. Backer, 'Finite element free surface flow analysis: a new tool for foundry engineers', in Proc. $6^{\text {th }}$ Int. Conf. on Modeling of Casting, Welding and Advanced Solidification Processes, T.S. Piwonka, V. Voller and L. Katgerman (eds.), The Minerals Metals and Materials Society, 405-412 (1993)

2. A. Bourg, A. Latrobe, P. Laty, G. Kreziak and C. Rigaut, 'Predicting the mould filling time with Simulor', in Proc. NUMIFORM'92 Int. Conf. on Numerical Modelling of Forming Processes, J.L.Chenot, R.D.Wood and O.C.Zienkiewicz (eds.), Balkema, Rotterdam, 777-785 (1992)

3. R. Codina, U. Schäfer and E. Onate, 'Mould filling simulation using finite elements', Int. J. Num. Meth. Heat Fluid Flow, 4, 291-310 (1994).

4. R.W. Lewis, A.S. Usmani and J.T.Cross, 'An efficient finite element method for mould filling simulation in metal casting', Num. Meth. in Thermal Problems, 7, 273-283 (1993)

5. R.W. Lewis, A.S. Usmani and J.T. Cross, 'Efficient mould filling simulation in castings by an explicit finite element method', Int. J. Num. Meth. Fluids, 20, 493-506 (1995)

6. F. Mampaey and Z.A. Xu, 'Simulation and experimental validation of mould filling', in Proc. $7^{\text {th }}$ Int. Conf. on Modeling of Casting, Welding and Advanced Solidification Processes, M. Cross and J. Campbell (eds.), The Minerals Metals and Materials Society, 3-14 (1995)

7. M. Médale and M. Jaeger, 'Numerical simulation of incompressible flows with moving interfaces', Int. J. Num. Meth. Fluids, 24, 615-638 (1997)

8. D.M. Waite and M.T. Samonds, 'Finite element free surface modeling', in Proc. $6^{\text {th }}$ Int. Conf. on Modeling of Casting, Welding and Advanced Solidification Processes, T.S. Piwonka, V. Voller and L. Katgerman et al. (eds.), The Minerals Metals and Materials Society, 357-364 (1993)

9. J.D. Zhu and I. Ohnaka, 'Computer simulation of fluid flow and heat transfer in partially reinforced casting', in Proc. 7th Int. Conf. on Modeling of Casting, Welding and Advanced Solidification Processes, M. Cross and J. Campbell (eds.), The Minerals Metals and Materials Society, 23-30 (1995)

10. C.W. Hirt and B.D. Nichols, 'Volume of fluid (vof) method for the dynamics of free boundaries', J. Comp. Phys., 39, 201-225 (1981) 
11. E. Thompson, 'Use of pseudo-concentration to follow creeping viscous flows during transient analysis', Int. J. Num. Meth. Fluids, 6, 749-761 (1986)

12. F.H. Harlow and J.E. Welch, "Numerical calculation of time-dependent viscous incompressible flow of fluid with free surface', Int. Phys. Fluids, 8, 2182-2189 (1965)

13. C.W. Chen, H.Y. Chou, C.R. Li, C.T. Shei and W.S. Hwang, 'Comparison of different computational dynamics techniques on their applications to the modelling of mould filling in casting', Trans. Japan Foundrymen's Society, 12, 94-106 (1993)

14. L. Cheng and S. Armfield, 'A simplified marker and cell method for unsteady flows on non staggered grids', Int. J. Num. Meth. Fluids, 21, 15-34 (1995)

15. K. Zaidi, B. Abbes and C. Teodosiu, 'Finite element simulation of mold filling using marker particles and the $k-\varepsilon$ model of turbulence', Comput. Meth. Appl. Mech. Engrg., 144, $227-233(1997)$

16. S.E. Navti, K. Ravindran, C. Taylor and R.W. Lewis, 'Finite element modelling of surface tension effects using a Lagrangian-Eulerian kinematic description', Comput. Meth. Appl. Mech. Engrg., 147, 41-60 (1997)

17. F. Muttin, T. Coupez, M. Bellet and J.L. Chenot, 'Lagrangian finite element analysis of time dependent viscous free surface flow using an automatic remeshing technique. Application to metal casting flow', Int. J. Num. Meth. Engng., 36, 2001-2015 (1991)

18. C.W. Hirt, A.A. Amsden and J.L. Cook, 'An arbitrary Lagrangian-Eulerian finite element method for all flow speeds', J. Comp. Phys., 14, 227-253 (1974)

19. J. Donéa, P. Fasolli-Stella, S. Giuliani, J.P. Halleux and A.V. Jones, 'An arbitrary Lagrangian-Eulerian finite element method for transient dynamic fluid structure interaction problems', in Proc. SMIRT-5 Conf. Berlin, 1-10 (1979)

20. W.K. Liu, T. Belytschko and H. Chang, 'An arbitrary Lagrangian-Eulerian finite element method for path-dependent materials', Comput. Meth. Appl. Mech. Engrg., 58, 227$245(1986)$

21. D.J. Benson, 'An efficient, accurate, simple, ALE method for non linear finite element programs', Comput. Meth. Appl. Mech. Engrg., 72, 305-350 (1989)

22. P.J.G. Schreurs, F.E. Veldpaus and W.A.M. Brekelmans, 'Simulation of forming processes using the arbitrary Lagrangian-Eulerian formulation', Comp. Meth. Appl. Mech. Engrg., 58, 19-36 (1986) 
23. B. Magnin, 'Modélisation du remplissage des moules d'injection pour polymères thermoplastiques par une méthode eulérienne lagrangienne arbitraire (Modelling of the filling of injection moulds for thermoplastic polymers by an arbitrary Eulerian Lagrangian method)', PhD Thesis, in french, Ecole des Mines de Paris (1994)

24. J. Donéa and L. Quartapelle, 'An introduction to finite element methods for transient advection problems', Comp. Meth. Appl. Mech. Engrg., 95, 169-203 (1992)

25. A.N. Brooks and T.J.R. Hughes, 'Streamline upwind / Petrov-Galerkin formulations for convection dominated flows with particular emphasis on the incompressible Navier-Stokes equations', Comp. Meth. Appl. Mech. Engrg., 32, 199-259 (1982)

26. O. Pironneau, Méthodes des Eléments Finis pour les Fluides (Finite Element Method for Fluid Flow), in french, Masson, Paris, 1988.

27. S. Ghosh and N. Kikuchi, 'An arbitrary Lagragian-Eulerian finite element method for large deformation analysis of elastic-viscoelastic solids', Comp. Meth. Appl. Mech. Engrg., 86, 127-188 (1991)

28. R.W. Lewis, S.E. Navti and C. Taylor, 'A mixed Lagrangian-Eulerian approach to modelling fluid flow during mould filling', Int. J. Num. Meth. Fluids, 25, 931-952 (1997)

29. L. Gaston, B. Glut, M. Bellet and J.L. Chenot, 'An arbitrary Lagrangian Eulerian finite element approach to non steady state fluid flows. Application to mould filling', in Proc. $7^{\text {th }}$ Int. Conf. on Modeling of Casting, Welding and Advanced Solidification Processes, M. Cross and J. Campbell (eds.), The Minerals Metals and Materials Society, 163-170 (1995)

30. L. Gaston, M. Bellet and J.L. Chenot, 'An arbitrary Lagrangian-Eulerian finite element approach to non steady state fluid flows. Application to mould filling', in Proc. ECCOMAS'96, 2nd ECCOMAS (European Community on Computational Methods in Applied Sciences) Computational Fluid Dynamics Conf., J.A. Désidéri et al. (eds.), John Wiley and Sons, Chichester, 973-979 (1996)

31. R. Schistel, Modélisation et Simulation des Ecoulements Turbulents (Modelling and Simulation of Turbulent Flows), in french, Hermès, Paris, 1993.

32. B.E. Launder and D.B. Spalding, Lectures in Mathematical Models of Turbulence, $3^{\text {rd }}$ edn, Academic Press, London, 1979.

33. I.L. Ryhming, Dynamique des Fluides (Fluid Dynamics), in french, $2^{\text {nd }}$ edn, Presses Polytechniques Universitaires Romandes, Lausanne, 1991. 
34. D. Elkaïm, Simulation numérique d'écoulements turbulents avec réactions chimiques (Numerical simulation of turbulent flows with chemical reactions), $\mathrm{PhD}$ Thesis, in french, Ecole Polytechnique de Montréal, 1990.

35. L. Gaston, Simulation numérique par éléments finis bidimensionnels du remplissage de moules de fonderie et étude expérimentale sur maquette hydraulique (Bidimensional finite element numerical simulation of the filling of casting moulds and experimental study on a water model), Ph.D Thesis, in french, Ecole des Mines de Paris, 1997.

36. B. Koobus, Algorithmes multigrilles et algorithmes implicites pour les écoulements compressibles turbulents (Multigrid algorithms and implicit algorithms for compressible turbulent flows), Ph.D. Thesis, in french, Université de Nice-Sophia-Antipolis, 1994.

37. M. Fortin and A. Fortin, 'Experiments with several elements for viscous incompressible flows', Int. J. Num. Meth. Fluids, 5, 911-928 (1985)

38. F. Brezzi, 'On the existence, uniqueness and approximation of saddle-point problems arising from Lagrange multipliers', RAIRO, R2, 129-151 (1974)

39. M. Fortin and R. Glowinski, Méthodes de Lagrangien Augmenté (Augmented Lagrangian Methods), in french, Dunod, Collection Méthodes Mathématiques de l'Informatique, 1982.

40. D. Pelletier, A. Garon and F. Ilinca, 'Adaptive finite element algorithms for the $\mathrm{k}-\mathcal{E}$ and k- $\omega$ models of turbulence', ASME Advances in Finite Element Analysis in Fluid Dynamics, 200, 59-68 (1994)

41. T. Coupez, Grandes déformations et remaillage automatique (Large deformations and automatic remeshing), Ph.D Thesis, in french, Ecole des Mines de Paris, 1991.

42. B. Glut, T. Coupez and J.L. Chenot, 'Automatic mesh generator for complex 2D domains - application to moving geometries in forming processes', J. of Mat. Proc. Tech., 34, 69-76 (1992)

43. J.P. Benqué, B. Ibler, A. Keramsi and G. Labadie, 'A new finite element method for the Navier-Stokes equations coupled with a temperature equation', in Proc. $4^{\text {th }}$ Int. Symp. on Finite Element in Flow Problem, T. Kawai (ed.), North-Holland, 295-301 (1982)

44. R.V. Westphal, J.P. Johnston and J.K. Eaton, 'Experimental study of flow reattachment in a single-sided sudden expansion', NASA contract report 3765, 1984. 
45. M. Jaeger and G. Dhatt, 'An extended $k$ - $\varepsilon$ finite element model', Int. J. Num. Meth. Fluids, 14, 1325-1345 (1992)

46. A.P. Gonze, Méthode directe de résolution d'écoulements turbulents discrétisés par les éléments finis (Direct method of resolution of turbulent flows discretised by finite elements), $\mathrm{PhD}$ Thesis, in french, Université catholique de Louvain, Louvain-la-Neuve, 1994. 


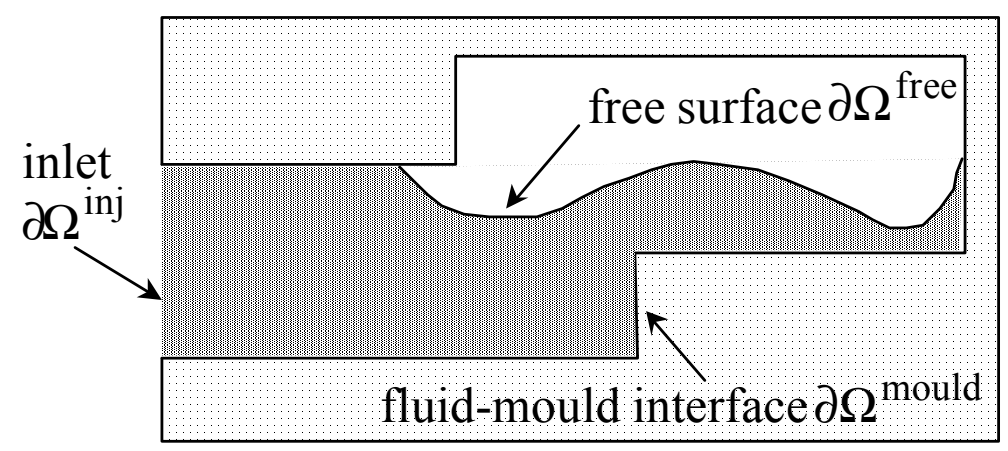

Figure 1: Boundary conditions for mould filling (schematic).

- velocity

$\square \rightarrow$ pressure



Figure 2: P2+/P1 finite element. 


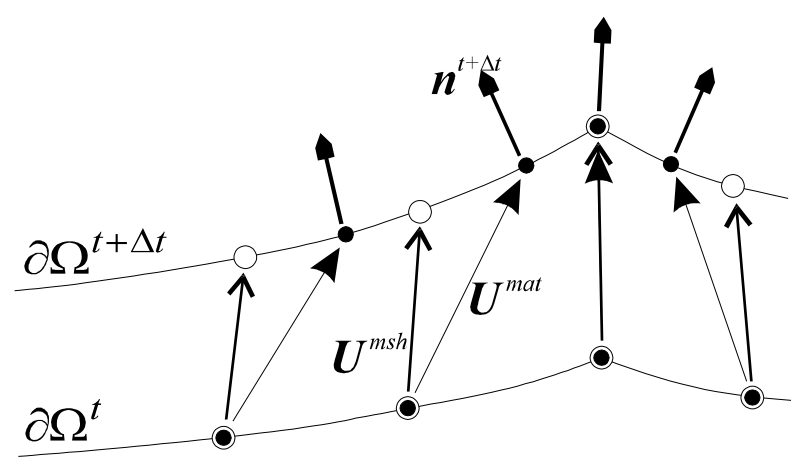

\begin{tabular}{|ll|} 
& $\stackrel{\boldsymbol{U}^{\text {mat }}}{\longrightarrow} \bullet$ particle \\
Legend: & $\stackrel{\boldsymbol{U}^{m s h}}{\longrightarrow}$ node \\
& $\stackrel{\boldsymbol{n}^{t+\Delta t}}{\longrightarrow}$ \\
\hline
\end{tabular}

Figure 3: Free surface mesh regularisation. 


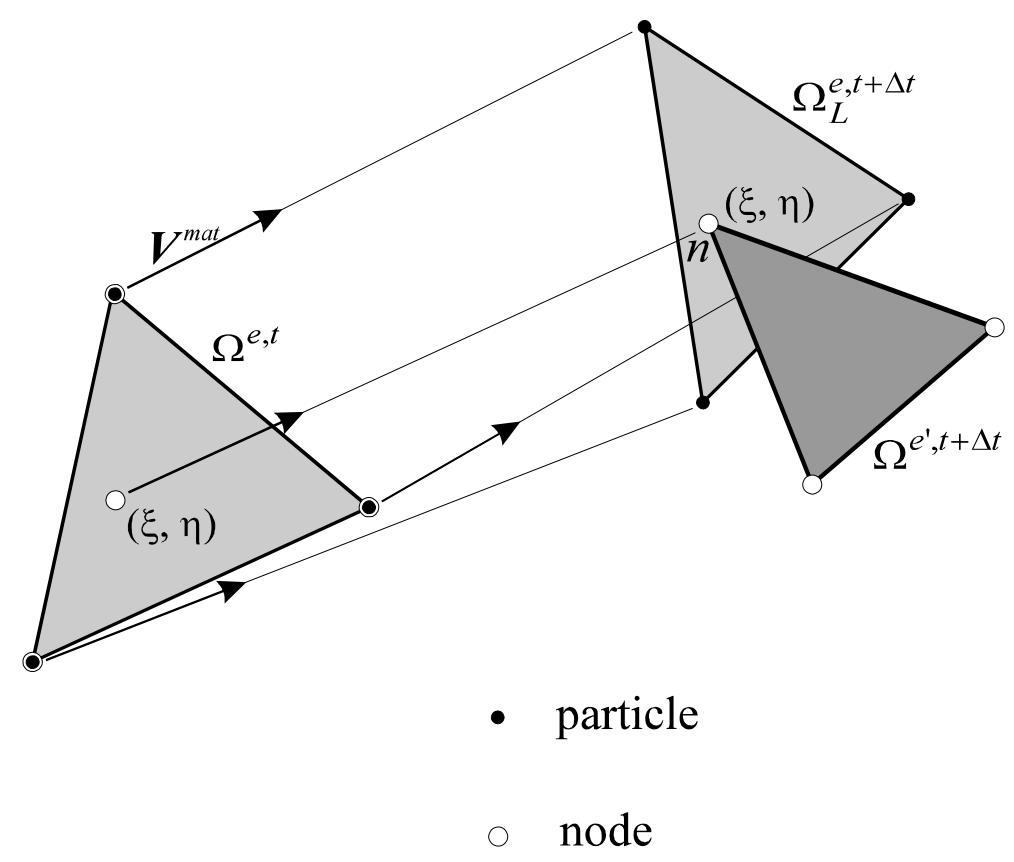

Figure 4: Illustration of transport procedure by use of a pseudo Lagrangian update and direct interpolation. 


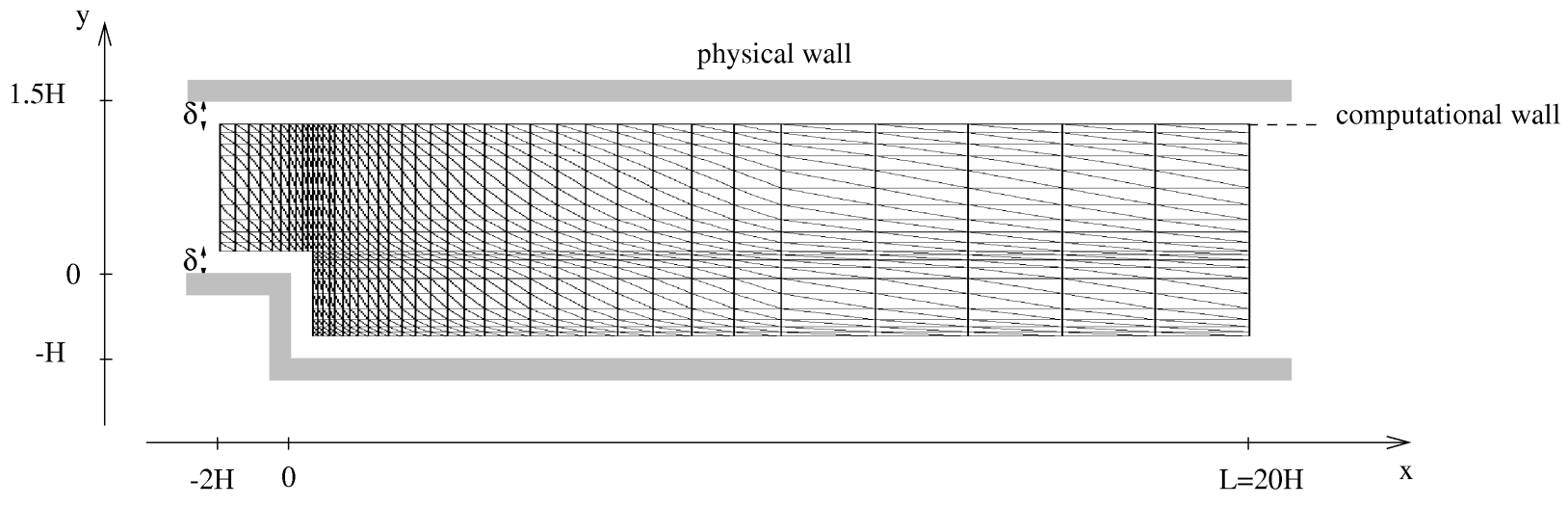

Figure 5: Configuration of BFS test and finite element mesh used for the computation (the value of $\delta$ has been magnified). 

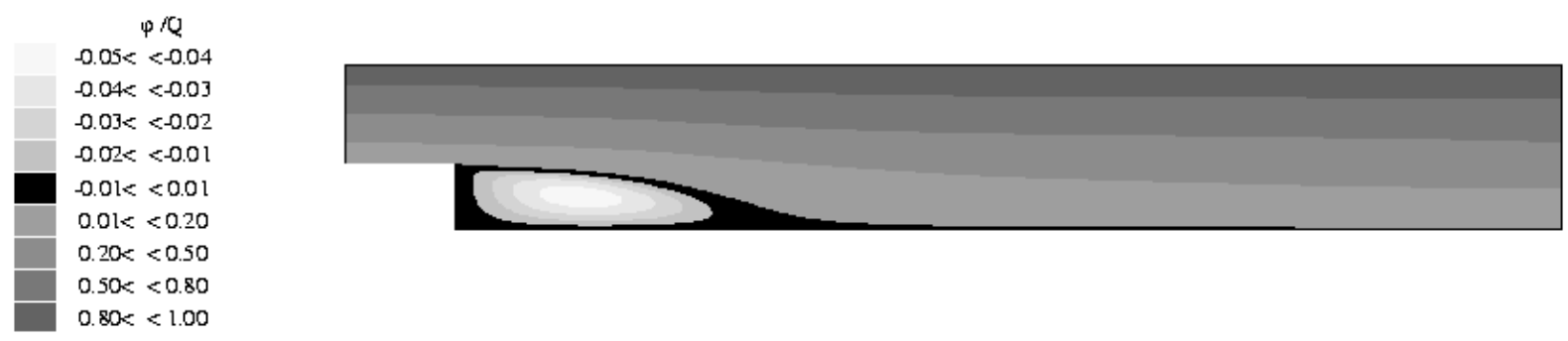

Figure 6: BFS test. Computed stream lines at convergence (iso-values of the stream function $\varphi$ normalized with the flow rate $Q$ ). 

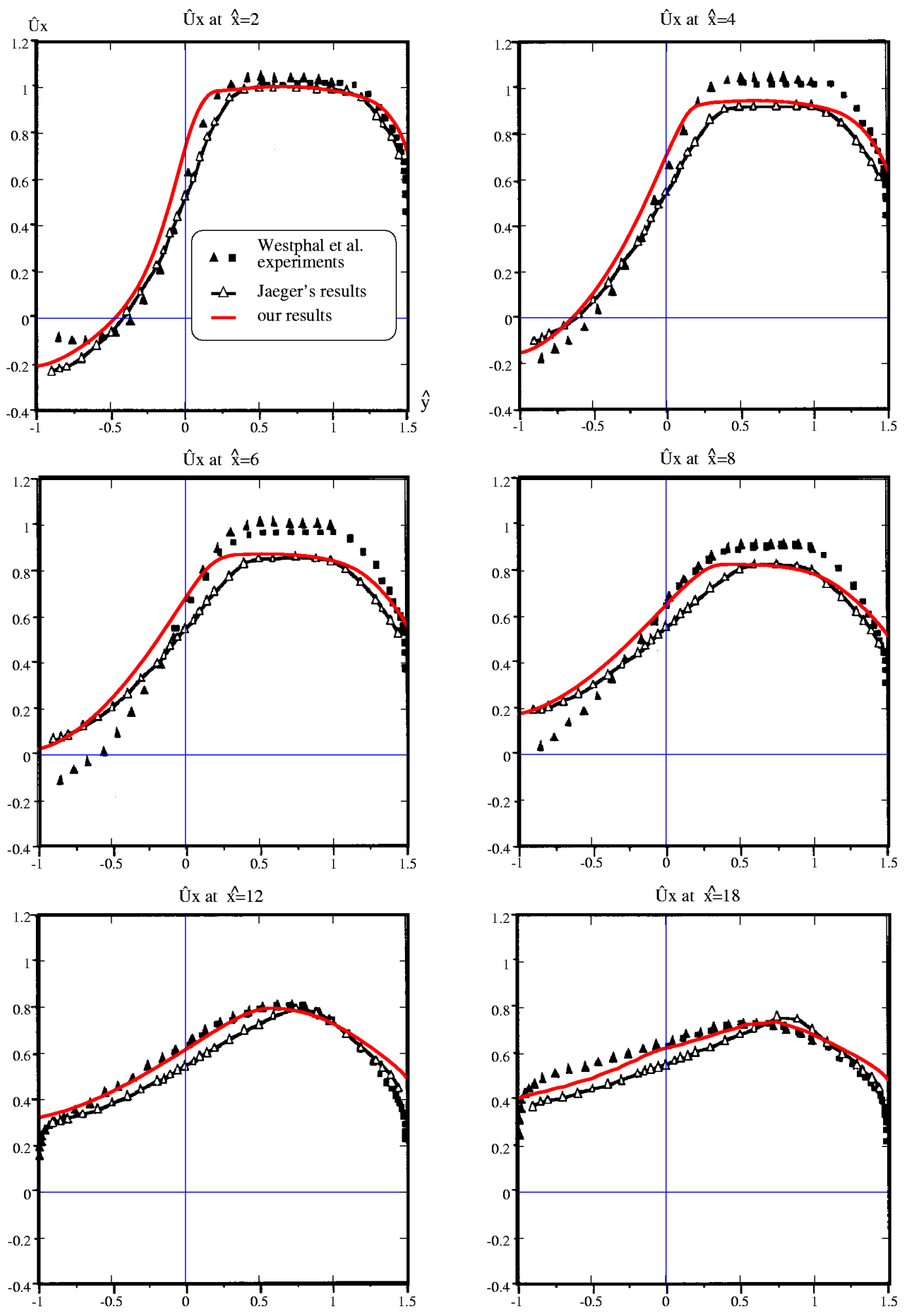

Figure 7: BFS test. Axial velocity profile in different transverse sections located at various horizontal coordinates. On each graphic, the normalised horizontal velocity $\hat{U}_{x}=v_{x} / V_{a v}$ is plotted versus the normalised vertical coordinate $\hat{y}=y / H$. The six graphics correspond to six transverse sections located at various normalised horizontal coordinates $\hat{x}=x / H$. 

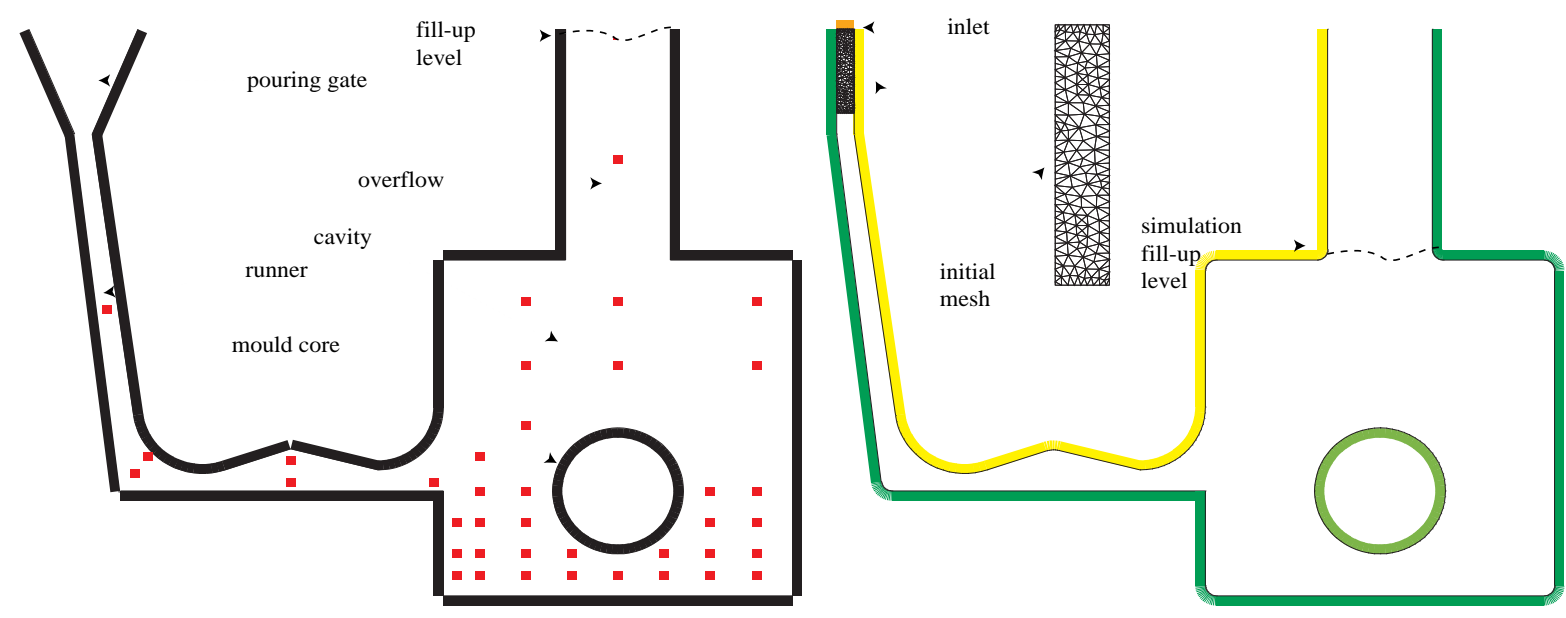

(a)

(b)

Figure 8: (a): Geometry of the mid-section of the experimental mould, with location of contact sensors. The square cavity is $160 \times 160 \mathrm{~mm}$. The thickness of the cavity in the normal direction is $8 \mathrm{~mm}$. (b): Two-dimensional configuration used for the simulation. 

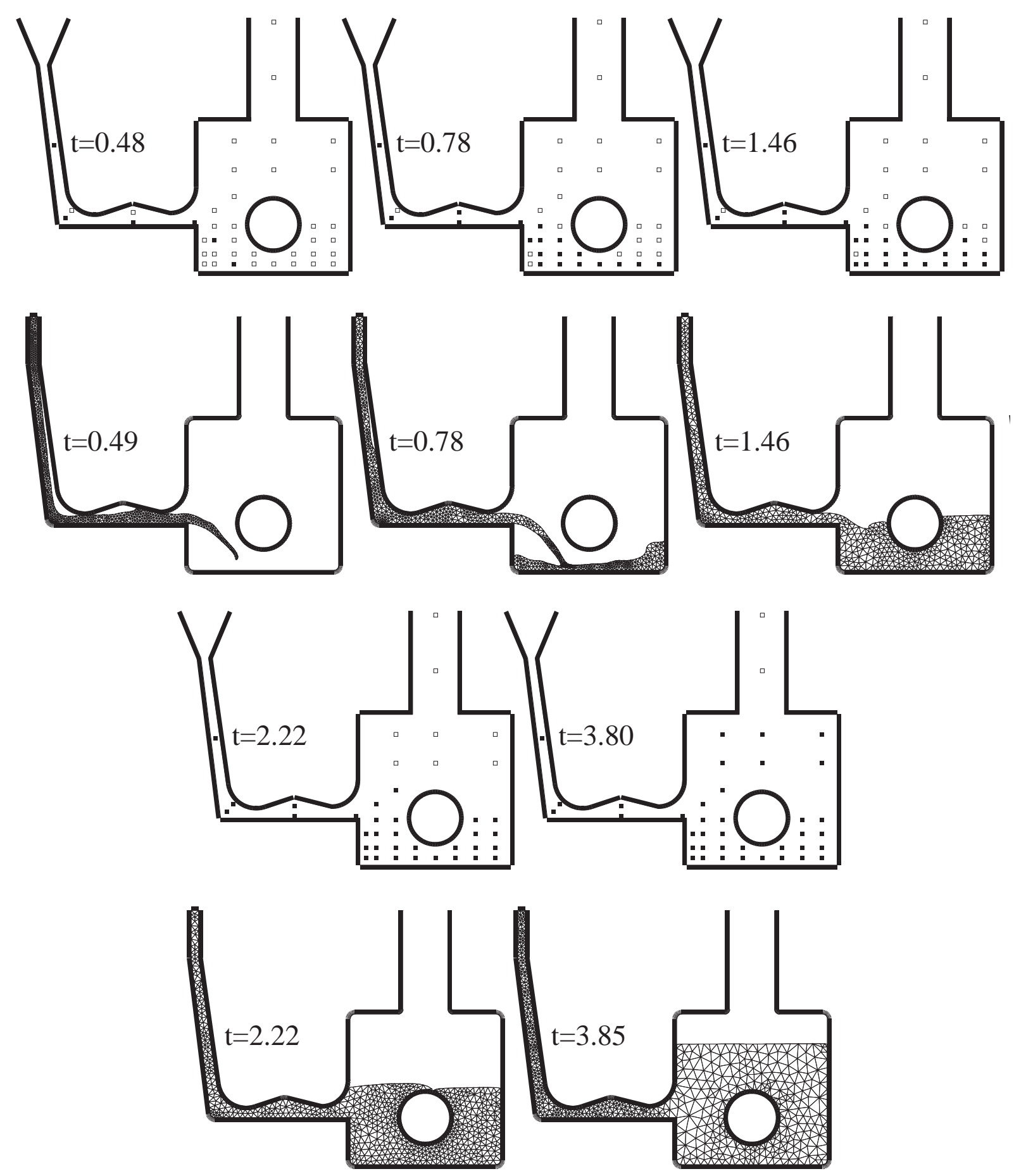

Figure 9: Progress of metal flow in the mould at different filling times. Lines 1 and 3 show the activated (black filled squares) and non activated (empty squares) contact sensors. Lines 2 and 4 show the finite element mesh at same filling times. 


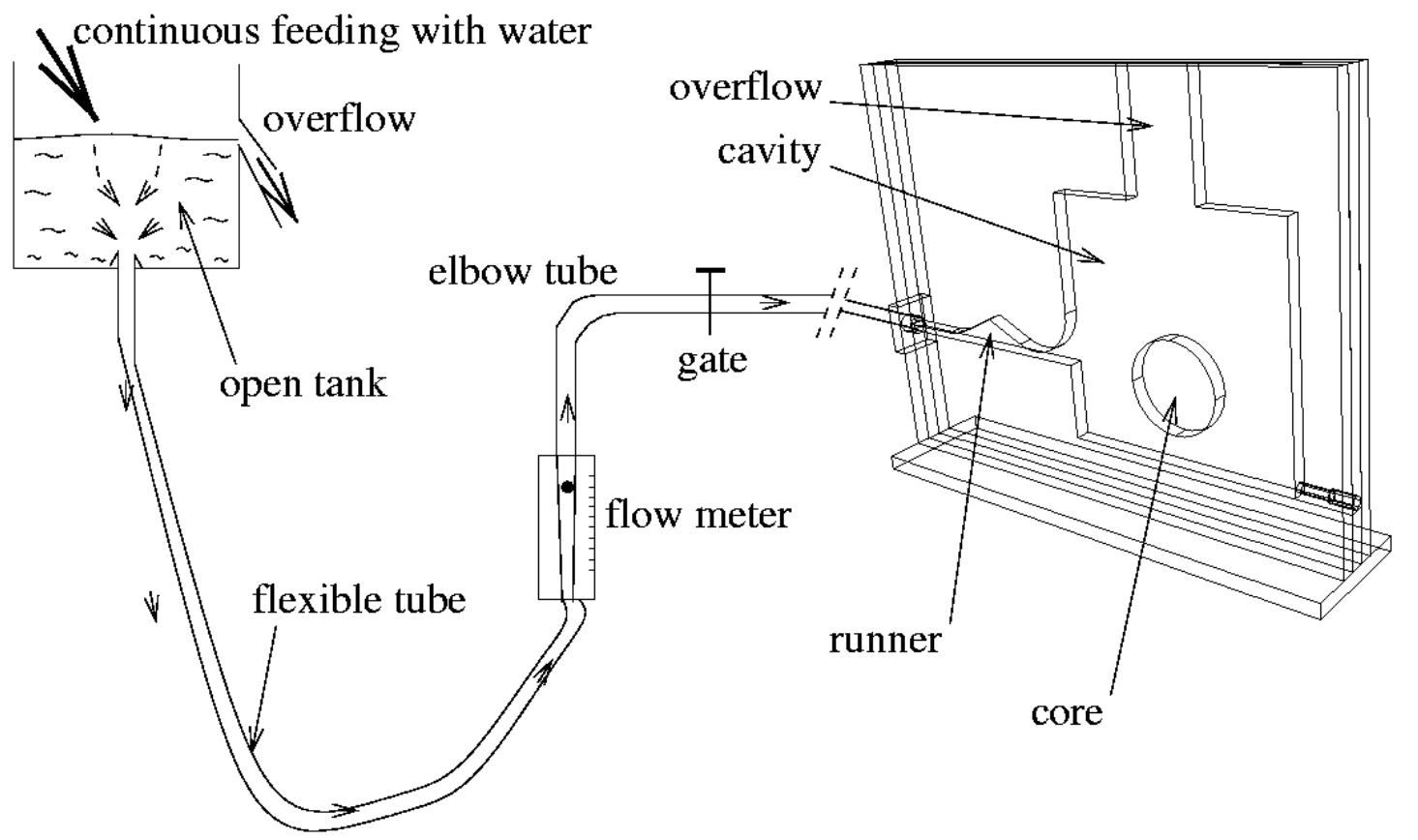

Figure 10: Water model. Experimental set-up. 

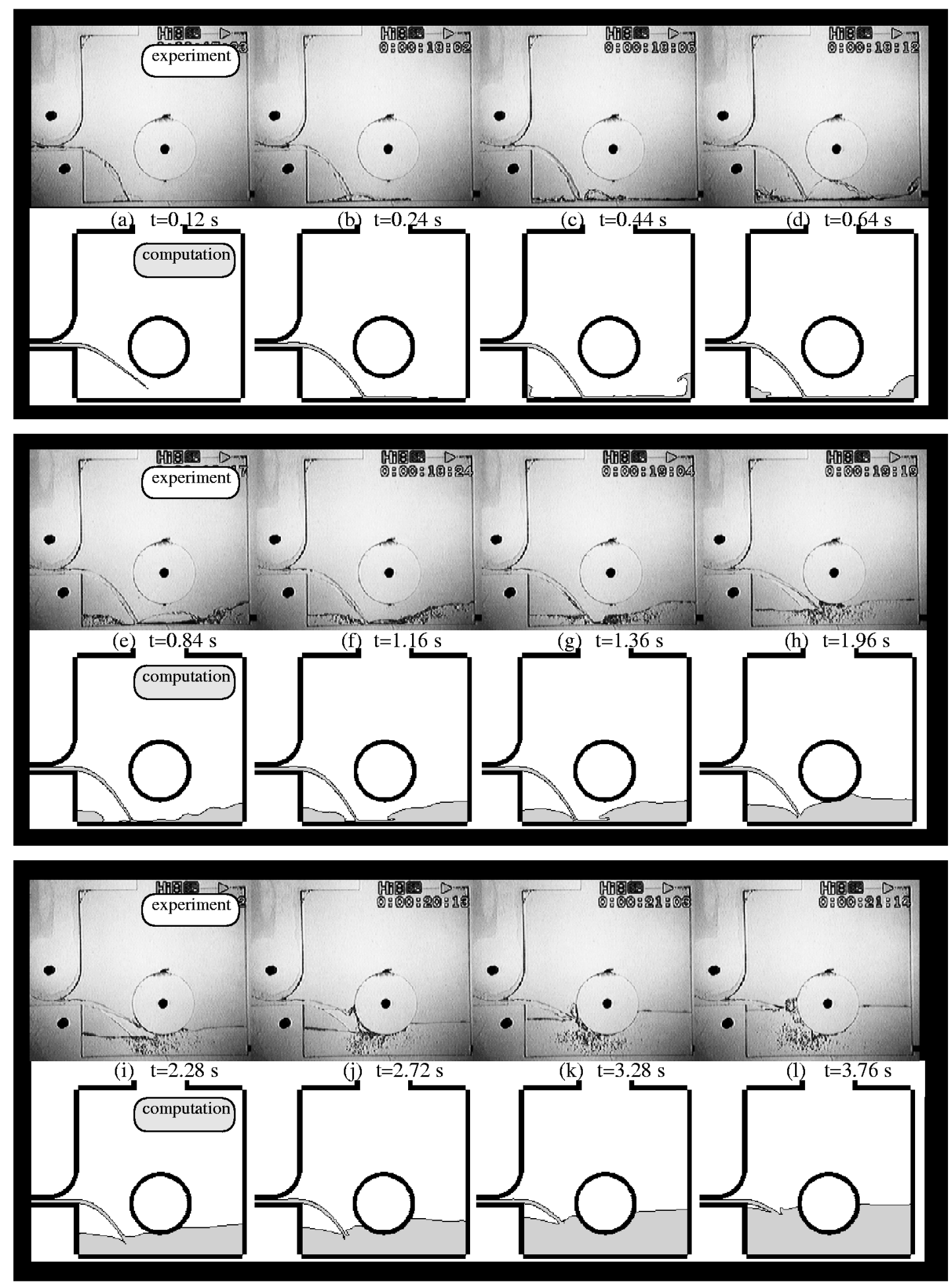

Figure 11a: Water model. Comparison between experimental flow and turbulent finite element computation. 

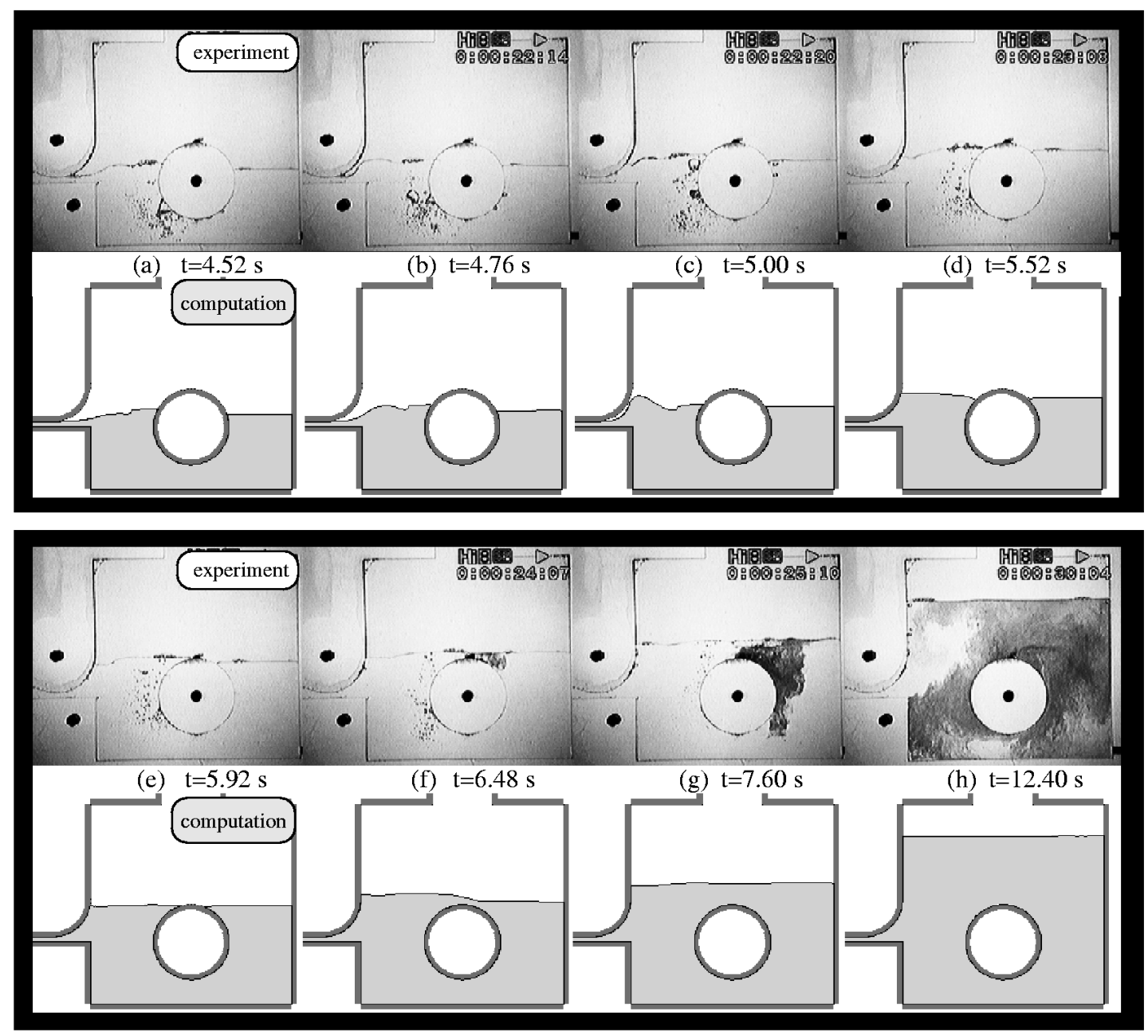

Figure 11b: Water model. Comparison between experimental flow and turbulent finite element computation. 


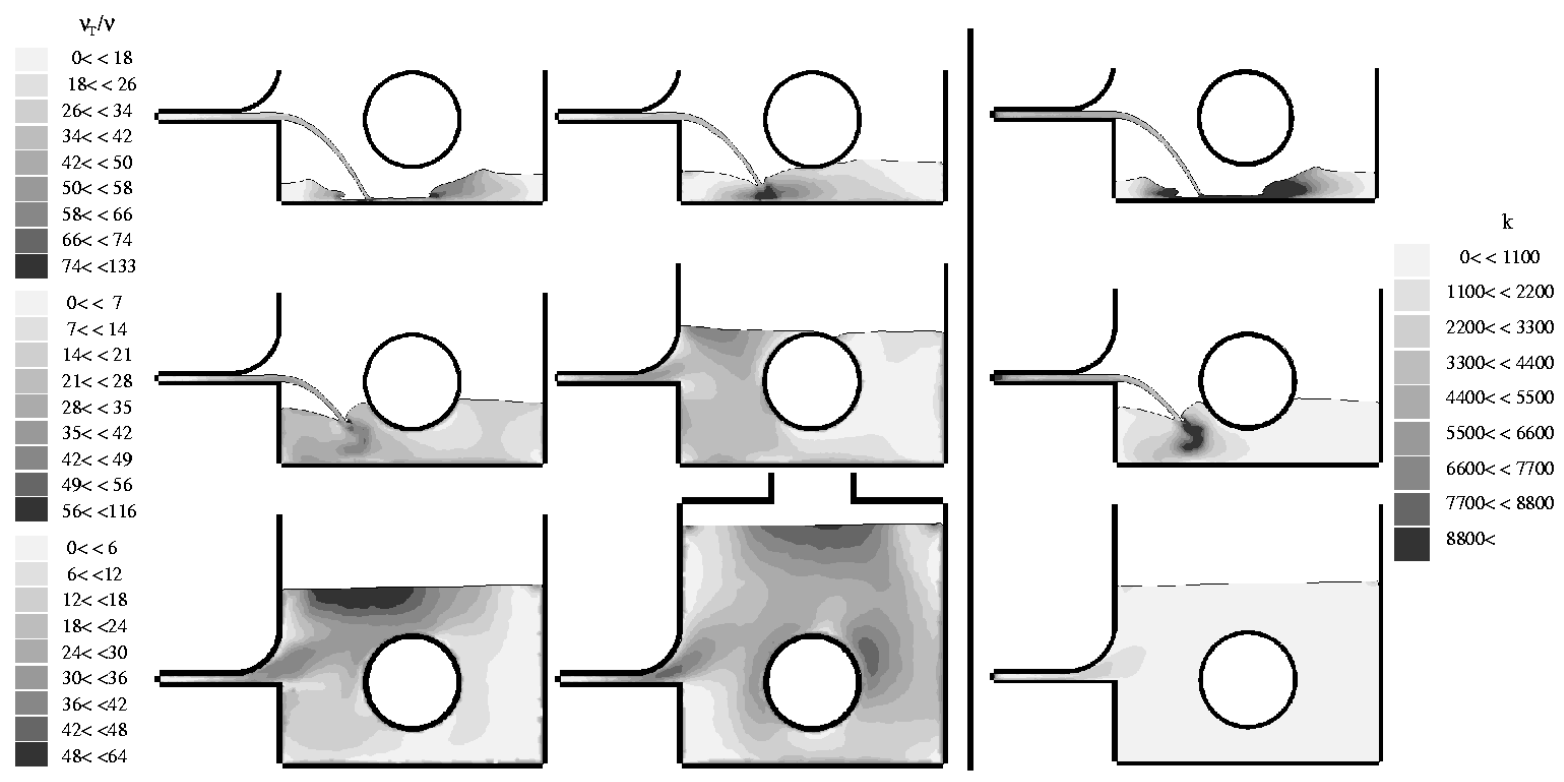

Figure 12: Water model. Left: Isovalues of the ratio between the turbulent and the nominal kinematic viscosities $v_{T} / v$. Right: computed distribution of the turbulent kinematic energy $k$. 